\title{
MASTER
}

\section{ISC origin times for announced and presumed underground nuclear explosions at several test sites}

Howard C. Rodean

December 3, 1979 


\section{ISC ORIGIN TIMES FOR ANNOUNCED AND PRESUMED UNDERGROUND NUCLEAR EXPLOSIONS AT SEVERAL TEST SITES}

\section{ABSTRACT}

Announced data for U.S. and French underground nuclear explosions indicate that nearly all detonations have occurred within one or two tenths of a second after the minute. This report contains ISC origin-time data for announced explosions at two U.S. test sites and one French test site, and includes similar data for presumed underground nuclear explosions at five Soviet sites. Origin-time distributions for these sites are analyzed for those events that appeared to be detonated very close to the minute. Particular attention is given to the origin times for the principal U.S. and Soviet test sites in Nevada and Eastern Kazakhstan, respectively. The mean origin times for events at the several test sites range from $0.4 \mathrm{~s}$ to $2.8 \mathrm{~s}$ before the minute, with the earlier mean times associated with the Soviet sites and the later times with the U.S. and French sites. These times indicate lower seismic velocities beneath the U.S. and French sites, and higher velocities beneath the sites in the U.S.S.R.

\section{INTRODUCTION}

The $m_{b}: M_{s}$ relation for explosiuns at the Nevada Test Site (NTS) differs from that for explosions in most other parts of the world. Evidence indicates that this difference results mostly from thigh body-wave attenuation in the upper mantle beneath the western U.S. Marshull, Springer, and Rodean (1979) developed an empirical magnitude for bodywave attenuation in the upper mantle and applied it to both source (explosion) and receiver (seismometer) ends of the teleseismic body-wave path. Their magnitude correction is an empirical function of the $P_{n}$-wave velocity (upper-mantle superficial wave velocity). Marshall et al. assumed a strong correlation between seismic absorption in the upper mantle and the $P_{n}$ velocity. In other words, their working hypotheses were that a relationship exists between $P$-wave velocity and $Q$ in the upper mantle, and that the $P_{n}$ velocity represents the quality of the upper mantle. They used $P_{r}$ velocity data to develop magnitude corrections for 87 receiver (seismograph) and 10 source (explosion) locations. They designated their body-wave magnitude as $m_{Q}$ to distinguish it from other definitions of $m_{b}$. Among their results, they showed that the $m_{b}: M_{s}$ data for NTS explosions are anomalous with respect to the data for explosions in other parts of the world, while the $m_{Q}: M_{s}$ data are much less so.

The evidence considered by Marshall, et al. includes observations by many investigators that, in regions where observed $\mathrm{P}$-wave amplitudes are generally low, $P_{n}$ velocities tend to be low and $P$ wave arrivals are generally late. In their paper, Marshall et al. presented their correlations between $P_{n}$ velocities and $P$-wave amplitude r'siduals as well as between $P_{n}$ velocities and $P$-wave transit-time residuals in the main body and Appendix B, respectively. Their correlations were for the receiver (seismograph) locations; in this study the correlation between $P_{n}$ velocity and travel time, as indicated by calculated event origin time, for source (explosion) locations is examined.

In this study, the ISC origin times for announced or probable underground nuclear explosions at two U.S., one French, and five Soviet test sites are analyzed. The data sets range from three explosions each at one U.S. and one Soviet test site, to 217 and 129 explosions at the principal U.S. and Soviet test sites, respectively.

The following discussion shows that the ISC origin times for events vary significantly from test 
site to test site. The best statistical data are for the principal U.S. and Soviet test sites: the ISC origin times for NTS events tend to be less than one second before the minute while those for the principal Soviet test site in Eastern Kazakhstan tend to be about two seconds before the minute. Official an- nouncements by the U.S. and France indicate that nearly all of their underground nuclear tests were conducted within one or two tenths of a second after the minute. It appears likely that the Soviets follow the same practice.

\section{LOCATIONS OF TEST SITES}

The locations of the test sites considered in this study may be found in the Times Atlas (1977). The principal U.S. test site, NTS, is approximately $150 \mathrm{~km}$ northwest of the city of Las Vegas. Three of the testing areas within NTS are identified in Plate III of the Times Atlas: Frenchman Flat, Yucca Flat, and Pahute Mesa. Three U.S. tests have been conducted on Amchitka Island in the Aleutians (Plate 113). The French have announced thirteen tests in the Sahara near En Ekker, approximately $150 \mathrm{~km}$ north of the city of Tamanrasset (Plate 88). The principal Soviet test site is approximately $150 \mathrm{~km}$ west-southwest of the city of Semipalatinsk in Eastern Kazakhstan, and is identified as the
Nuclear Testing Ground (NTG) in Plate 43. The Soviets have conducted their highest-yield tests (based on seismic magnitude data) on Novaya Zemlya (Plate 41). Most of these tests have been in the vicinity of the Matochkin Shar, a narrow strait that separates the northern and southern parts of the island. Some have been conducted at the southern tip of Novaya Zemlya near the village of Krasino. The Soviets have conducted a series of tests near the village of Azgir in the Ryn Peski, a sandy desert in Western Kazakhstan north of the Caspian Sea (Plate 45). Three Soviet tests have been conducted east of the Caspian Sea in the Ustyurt Plateau in Southwestern Kazakhstan (Plate 43).

\section{SOURCES OF DATA}

The source of seismic data for this study is the International Seismological Centre (ISC) which publishes a monthly Bulletin and a semi-annual Regional Catalogue of Eorthquakes. Both publications indicate if an event is probably an explosion; the Regional Catalogue includes a list of probable explosions.

The U.S. and France have announced data for many of their underground nuclear explosions. In this study, the data for U.S. explosions are from
Springer and Kinnaman $(1971,1975)$, and the data for French explosions are from Duclaux and Michoud (1970). The Soviet Union has not r.leased any data that could be used in this study. Therefore, the publications of Nordyke (1975) and Dahlman and Israelson (1977) were used together with ISC data in locating sites at which series of events (presumed underground nuclear explosions) have occurred.

\section{ANNOUNCED OR PRESUMED EXPLOSIONS}

The first semi-annual Regional Catalogue of Earthquakes published by the ISC was for the period of January through June 1964. The Regional Catalogue includes a list of probable explosions that is very convenient for use in studies of explosion seismology. Therefore, the data sets for U.S. and
Soviet explosions begin in 1964. The set for the U.S. extenos through 1973, the last year covered by Springer and Kinnaman. The set for the U.S.S.R. extends through 1976, the last year for which ISC data were available. Duclaux and Michaud presented data for 13 French underground tests in the 
Sahara during 1961-1966; ISC publications for this period contain data for only five of these events.

Announced and ISC data are presented in Tabie 1 for U.S. tests at the NTS, and in Table 2 for U.S. tests on Amchitka. Announced and ISC data for French tests in the Sahara are given in Table 3. ISC data for presumed Soviet tests at the NTG, on Novaya Zemlya, near Azgir, and on the Ustyurt Plateau are given in Tables 4 through 7, respectively.
As indicated in Table 5, two events near Krasino at the sourthern end of Novaya Zemlya on 740707 and 740722 are not included because the ISC identified them as probable aftershocks of the event on 731007. Israelson et al. (1974) reported a series of ten aftershocks within four houtrs after the main event of 731007.

TABLE 1. Announced and ISC data for 217 U.S. tests at NTS from 1964 to 1973 . These testa are listed in both the data summaries by Springer and Kinnaman and the ISC publications. The locations of these teats are centered at approximately $37^{\circ} \mathrm{N}-116^{\circ} \mathrm{N}$.

\begin{tabular}{|c|c|c|c|c|c|c|}
\hline \multirow[b]{2}{*}{ Date } & \multirow[b]{2}{*}{ Name } & \multicolumn{2}{|c|}{ Time, GMT (h-m-s) } & \multirow{2}{*}{$\begin{array}{c}\text { ISC } \\
\text { depth, km }\end{array}$} & \multirow{2}{*}{$\begin{array}{l}\text { No. obs. } \\
\text { to IBC }\end{array}$} & \multirow{2}{*}{$\begin{array}{l}\text { ISC } \\
m_{b}\end{array}$} \\
\hline & & Announced & ISC & & & \\
\hline 640116 & Fore & 160000.15 & $1.60002 .4 \pm 0.16$ & $25 \pm 6.6$ & 50 & 5.2 \\
\hline 640123 & Oconto & 160000.15 & $160001.8 \pm 0.29$ & $15 \pm 31$ & 20 & 4.2 \\
\hline 640220 & Klickitat & $1536 \quad 00.14$ & $153002.1 \pm 0.19$ & $20 \pm 8.1$ & 47 & 5.1 \\
\hline 640313 & Pike & 160200.12 & 160200.1 & $\mathbf{0}$ & 14 & - \\
\hline 640414 & Hook & $1440 \quad 00.12$ & $143959.7 \pm 0.21$ & $\mathbf{0}$ & 15 & - \\
\hline 640415 & Sturgeon & 143000.12 & $143006.6 \pm 2.0$ & $\mathbf{0}$ & 12 & - \\
\hline 640424 & Turf & 201000.16 & $201001.3 \pm 0.22$ & $17 \pm 9$ & 55 & 5.2 \\
\hline 640429 & Pipe Fish & $2047 \quad 00.12$ & $2047 n 0.1 \pm 0.39$ & $\mathbf{0}$ & 17 & 4.1 \\
\hline 640514 & Backswing & 144000.15 & $144000.1 \pm 0.28$ & $\mathbf{0}$ & 20 & - \\
\hline 640515 & Minnow & 161500.12 & $161459.6 \pm 0.55$ & $\mathbf{0}$ & 14 & - \\
\hline 640611 & Ace & 164500.15 & $164501 \pm 0.8$ & $\mathbf{0}$ & 12 & - \\
\hline 640625 & Fade & 133000.14 & $133000.3 \pm 0.25$ & $\mathbf{0}$ & 24 & - \\
\hline 640630 & Dub & 133300.14 & $133259.8 \pm 0.24$ & $-\quad \mathbf{0}$ & 20 & - \\
\hline 640716 & Bye & 131500.13 & $131459.9 \pm 0.30$ & $\mathbf{0}$ & 26 & - \\
\hline 640819 & Alve & 160000.14 & $160001.8 \pm 1.0$ & $\mathbf{0}$ & 14 & - \\
\hline 640822 & Canvesheck & 221700.06 & $221701.2 \pm 0.74$ & $B \pm 76$ & 22 & - \\
\hline 640828 & Haddock & 170600.04 & $170601.9 \pm 0.2$ & $16 \pm 19$ & 15 & - \\
\hline 640904 & Gumay & 181500.08 & $181501.8 \pm 0.14$ & $12 \pm 12$ & 24 & - \\
\hline 641002 & Auk & $2003 \quad 00.04$ & $200300.6 \pm 0.20$ & $4 \pm 10$ & 37 & 4 \\
\hline 641009 & Par & $1400 \quad 00.12$ & $140002.6 \pm 0.24$ & $20 \pm 14$ & 37 & 4.8 \\
\hline 641016 & Bartiel & 155930.04 & $155930.5 \pm 0.45$ & $5 \pm 38$ & 16 & - \\
\hline 641031 & Foreat & 170458.61 & $170458.0 \pm 0.22$ & 0 & 20 & - \\
\hline 641105 & Findear & 150000.11 & $150001.5 \pm 0.7: 0$ & $16 \pm 10$ & 34 & 4.8 \\
\hline 641205 & Crepe & 211500.10 & $211503.1 \pm 0.1 .9$ & $28 \pm 9$ & 46 & 4.8 \\
\hline 641216 & Mudpack & 211000.10 & $211000 \pm 1 .: ?$ & $\mathbf{0}$ & 16 & - \\
\hline 650114 & Wool & 160000.14 & $160000.7 \pm 0.16$ & $4 \pm 32$ & 22 & - \\
\hline 650204 & Cahmere & 153000.11 & $152959.7 \pm 0.20$ & $\mathbf{0}$ & 18 & - \\
\hline 650216 & Medin & 173000.04 & $173001.6 \pm 0.32$ & $13 \pm 52$ & 22 & - \\
\hline 650218 & Widhbone & 161847.15 & $161847.3 \pm 0.30$ & $5 \pm 22$ & 28 & - \\
\hline 650303 & Wugutil & 191300.03 & 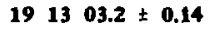 & $32 \pm 3$ & 77 & - \\
\hline 650326 & Cup & 153408.16 & $163410.6 \pm 0.14$ & $26 \pm 6$ & 64 & - \\
\hline 650405 & Kentrel & $2100 \quad 00.04$ & $210003.3 \pm 0.42$ & $29 \pm 26$ & 26 & - \\
\hline
\end{tabular}


TABLE 1. (Continued.)

\begin{tabular}{|c|c|c|c|c|c|c|}
\hline \multirow[b]{2}{*}{ Dute } & \multirow[b]{2}{*}{ Name } & \multicolumn{2}{|c|}{ Time, GMT (h-m-s) } & \multirow{2}{*}{$\begin{array}{c}\text { ISC } \\
\text { depth, km }\end{array}$} & \multirow{2}{*}{$\begin{array}{l}\text { No. abs } \\
\text { to IBC }\end{array}$} & \multirow{2}{*}{$\begin{array}{l}\text { ISC } \\
\mathbf{m}_{\mathbf{b}}\end{array}$} \\
\hline & & Announced & ISC & & & \\
\hline 650414 & Palanquin & 131400.11 & $131402.6 \pm 0.20$ & $20 \pm 15$ & 23 & \\
\hline 650421 & Gum Drop & 220000.03 & $220003.4 \pm 0.18$ & $35 \pm 8$ & 33 & 9.0 \\
\hline 650507 & Tee & 154711.15 & $154711.3 \pm 0.30$ & $\mathbf{0}$ & 20 & - \\
\hline 650512 & Buben & 181500.10 & 181500.0 & o & 18 & - \\
\hline 650514 & Scamp & 173236.23 & $173238.3 \pm 0.16$ & $20 \pm 8$ & 33 & - \\
\hline 650521 & Tweed & 13 of 52.11 & $130854.2 \pm 0.21$ & $30 \pm 12$ & 33 & - \\
\hline 650611 & Petrel & 194500.04 & $194500.4 \pm 0.56$ & $4 \pm 55$ & 14 & - \\
\hline 650616 & Diluted Waters & 163000.15 & $163000.0 \pm 0.35$ & $1 \pm 24$ & 26 & - \\
\hline 650723 & Bronze & 170000.04 & $170002.0 \pm 0.14$ & $18 \pm 6$ & 100 & 5.4 \\
\hline 650806 & Mauve & 172330.04 & $172330.8 \pm 0.28$ & $8 \pm 24$ & 27 & - \\
\hline 650901 & Sereimer & 20 ne 90.04 & $200739.4 \pm 0.26$ & o & 28 & 4.2 \\
\hline 650910 & Charcaal & 171200.03 & $171202.1 \pm 0.23$ & $22 \pm 10$ & 48 & 3.1 \\
\hline 651203 & Conturoy & 151302.10 & $151304.52 \pm 0.13$ & $25 \pm 6$ & 97 & 5.6 \\
\hline 651216 & Buff & 191500.04 & $191502.6 \pm 0.13$ & $27 \pm 5$ & 76 & 5.3 \\
\hline 660113 & Maxwell & $15 \quad 3743.10$ & $153743.0 \pm 0.33$ & o & 11 & - \\
\hline 660118 & Lampblock & $18 \quad 3500.04$ & $183503.3 \pm 3.26$ & $31 \pm 24$ & 35 & 5.2 \\
\hline 660121 & Dovekie & 182800.04 & $182759.3 \pm 0.15$ & o & 17 & - \\
\hline 660203 & Plnid II & $18 \quad 1737.10$ & $181737.1 \pm 0.34$ & o & 22 & - \\
\hline 660224 & Rex & $15 \quad 55 \quad 07.04$ & $155510.5 \pm 0.36$ & $35 \pm 6$ & 48 & 5.0 \\
\hline 660305 & Red Hot & $18 \quad 15 \quad 00.10$ & $181500.7 \pm 0.55$ & 0 & 18 & - \\
\hline 660307 & Pinfoot & 184100.07 & $184100.5 \pm 1.84$ & $\mathbf{0}$ & 7 & - \\
\hline 660312 & Claymer & $\begin{array}{lll}18 & 04 & 13.11\end{array}$ & $180413.2 \pm 0.26$ & $\mathbf{0}$ & 13 & - \\
\hline 660318 & Purple & 190000.04 & $190002.7 \pm 0.52$ & $\mathbf{0}$ & 29 & - \\
\hline 660401 & Lime & $1840 \quad 00.04$ & $184001.1 \pm 0.73$ & 0 & 12 & - \\
\hline 660406 & Sturz & $\begin{array}{lll}13 & 57 & 17.10\end{array}$ & $135719.9 \pm 1.29$ & $28 \pm 13$ & 32 & 4.4 \\
\hline 660407 & Tomutoe & $22 \quad 2730.04$ & $222733.4 \pm 1.46$ & $32 \pm 16$ & 22 & - \\
\hline 660414 & Duryen & 141343.10 & $141346.4 \pm 0.36$ & $34 \pm 4$ & $\mathbf{7 0}$ & 5.4 \\
\hline 660425 & Pin Stripe & $1838 \quad 00.14$ & $183803.3 \pm 0.94$ & $31 \pm 9$ & 39 & 4.5 \\
\hline 660504 & Traveler : & $\begin{array}{lll}13 & 32 & 17.09\end{array}$ & $133219 \pm 9.0$ & $11 \pm 70$ & 19 & - \\
\hline 660505 & Cyclemen & 140000.04 & $140003.1 \pm 1.13$ & $28 \pm 1$ & 29 & 4.4 \\
\hline 660506 & Chartreuse & 150000.08 & $150003.1 \pm 0.64$ & $29 \pm 5$ & 102 & 5.4 \\
\hline $6+5512$ & Tapestry & 193726.20 & $193726.1 \pm 3.24$ & $4 \pm 25$ & 27 & 4.7 \\
\hline 660513 & Piranha & $1330 \quad 00.04$ & $133002.1 \pm 0.65$ & $21 \pm 6$ & 101 & 3.4 \\
\hline 660519 & Dumont & $1356 \quad 28.14$ & $135630.6 \pm 0.63$ & $23 \pm 6$ & 144 & 5.9 \\
\hline 660527 & Discus Thrower & $2000 \quad 00.04$ & $200003 . \pm 0.91$ & $28 \pm 8$ & 32 & 5.0 \\
\hline 660602 & Ftle Driver & $\begin{array}{lll}15 & 30 & 00,09\end{array}$ & $153001.8 \pm 0.60$ & $18 \pm 4$ & 135 & 5.6 \\
\hline 660603 & Ten & 140000.04 & $140002.2 \pm 0.59$ & $22 \pm 4$ & 132 & 5.7 \\
\hline 660610 & Puce & $1430 \quad 00.04$ & $143002.6 \pm 1.53$ & $25 \pm 16$ & 25 & - \\
\hline 660625 & Vulcen & $\begin{array}{lll}17 & 13 & 00.07\end{array}$ & $171259.6 \pm 0.30$ & o & 31 & - \\
\hline 0.60630 & Helfbeak & 221500.07 & $221502.7 \pm 0.53$ & $23 \pm 4$ & 175 & 6.1 \\
\hline $660 \% 28$ & Baxon & 153330.13 & $153332.5 \pm 1.54$ & o & 15 & - \\
\hline 660810 & Roven: & 131600.07 & $131603 \pm 1.5$ & $\mathbf{0}$ & 12 & - \\
\hline 660912 & Detringer & $15 \quad 3000.54$ & $152959.8 \pm 0.25$ & $\mathbf{0}$ & 23 & 4.6 \\
\hline 660923 & Dequiri & $1800 \quad 00.04$ & $175958.3 \pm 0.32$ & $\mathbf{0}$ & 13 & - \\
\hline 660929 & Newark & $14 \quad 4530.09$ & $144532.1 \pm 1.10$ & 0 & 16 & - \\
\hline
\end{tabular}


TABLE 1. (Continued.)

\begin{tabular}{|c|c|c|c|c|c|c|}
\hline \multirow[b]{2}{*}{ Date } & \multirow[b]{2}{*}{ Name } & \multicolumn{2}{|c|}{ Time, GMT (h-m-1) } & \multirow{2}{*}{$\begin{array}{c}\text { ISC } \\
\text { depth, km }\end{array}$} & \multirow{2}{*}{$\begin{array}{l}\text { Nc. obn } \\
\text { to ISC }\end{array}$} & \multirow{2}{*}{$\begin{array}{l}\text { IBC } \\
\mathbf{m}_{\mathbf{b}}\end{array}$} \\
\hline & & Announced & ISC & & & \\
\hline 661103 & Simm: & $144500.00^{\circ}$ & $144501.2 \pm 0.63$ & $\mathbf{0}$ & 12 & - \\
\hline 661111 & Ajax & 120000.14 & $120000.7 \pm 0.57$ & 0 & 21 & - \\
\hline 661118 & Cerise & 150200.04 & 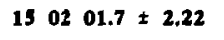 & $17 \pm 25$ & 25 & - \\
\hline 661213 & New Point & $2100 \quad 00,08$ & $210002.7 \pm 1.45$ & $23 \pm 15$ & 32 & 4.6 \\
\hline 661220 & Greeley & 153000.08 & $153001.9 \pm 0.54$ & $21 \pm 4$ & 231 & 6.3 \\
\hline 670119 & Nuch & 164500.14 & $164302.5 \pm 0.62$ & $25 \pm 3$ & 106 & 5.3 \\
\hline 670120 & Bourbon & $\begin{array}{lll}17 & 40 & 03.41\end{array}$ & $174005.3 \pm 0.56$ & $26 \pm 5$ & 79 & 5.3 \\
\hline 670208 & Ward & $15 \quad 1500.13$ & $151500.4 \pm 0.37$ & o & 23 & 4.6 \\
\hline 670223 & Persimmon & 183400.04 & $183400.5 \pm 0.89$ & $\mathbf{0}$ & 20 & 4.4 \\
\hline 670223 & Agile & $1850 \quad 00.0^{a}$ & $1850 \quad 02.9 \pm 0.55$ & $26 \pm 4$ & 162 & 5.6 \\
\hline 670302 & Rivet III & $150000.0^{2}$ & $150000.3 \pm 0.23$ & o & 31 & - \\
\hline 670407 & Fawn & 150000.04 & $150002.3 \pm 5.13$ & o & 10 & - \\
\hline 670421 & Chocalate & 150900,04 & $150904.4 \pm 0.44$ & $36 \pm 11$ & 23 & - \\
\hline 670427 & Effendi & $144500.0^{2}$ & $144502.8 \pm 8.85$ & $10 \pm 69$ & 24 & - \\
\hline 670510 & Mickey & $\begin{array}{lll}13 & 40 & 00.04\end{array}$ & $134003.0 \pm 0.70$ & $1 \pm 6$ & 35 & 4.9 \\
\hline 670520 & Commodore & $150000.0^{\star 2}$ & $150001.7 \pm 0.49$ & $16 \pm 3.8$ & 203 & 3.8 \\
\hline 670523 & Scoteh & 140000.04 & $140002.0 \pm 0.53$ & $20 \pm 4.1$ & 166 & 3.7 \\
\hline 670526 & Knickerbocker & $1500 \quad 01.50$ & $150005.1 \pm 0.25$ & $35 \pm 3$ & 117 & 5.4 \\
\hline 670622 & Switch & $131000.0^{2}$ & $131001.7 \pm 0.88$ & o & 21 & - \\
\hline 670626 & Midi Mist & $160000.0^{2}$ & $160002.2 \pm 0.74$ & $20 \pm 6.8$ & so & 5.1 \\
\hline 670629 & Umber & $\begin{array}{lll}11 & 25 & 00.04\end{array}$ & 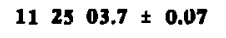 & $33 \pm 16$ & 25 & 4.6 \\
\hline 670727 & Stanley & $130000.0^{2}$ & $\begin{array}{lllll}13 & 00 & 02.7 & : 0.87\end{array}$ & $29 \pm 8.9$ & 43 & 5.0 \\
\hline 670810 & Washex & $141000.0^{x}$ & $141000.3 \pm 0.29$ & o & 17 & - \\
\hline 670818 & Bondenux & $2012 \quad 30.04$ & $201230.4 \pm 0.23$ & 3 & 34 & 4.6 \\
\hline 670831 & Door Mist & 163000.04 & $163000.4 \pm 0.45$ & o & 41 & 5.0 \\
\hline 670907 & Yard & $134300.0^{n}$ & $134503.1 \pm 0.86$ & $29 \pm 7.5$ & 73 & 5.0 \\
\hline 670921 & Marvel & $204500.0^{2}$ & $204504.1 \pm 1.39$ & $127 \pm 34$ & 17 & - \\
\hline 670927 & Zaze & $1700 \quad 00.04$ & $170002.4 \pm 0.48$ & $23 \pm 3.8$ & 192 & 3.7 \\
\hline 671018 & Lenpher & $143000.0^{2}$ & $143002.0 \pm 0.63$ & $19 \pm 8$ & 158 & 5.8 \\
\hline 671025 & Snzerac & 143000.06 & $143002.0 \pm 3.66$ & $9 \pm 29$ & 23 & - \\
\hline 671108 & Cobblex & 150000.04 & $150003.1 \pm 0.29$ & $32 \pm 4$ & 52 & 5.1 \\
\hline 671215 & Stilt & 130000.04 & $150003.5 \pm 1.46$ & $28 \pm 15$ & 32 & - \\
\hline 680118 & Hupmoblle & $163000.0^{a}$ & $163005.1 \pm 0.53$ & $35 \pm 16$ & 25 & - \\
\hline 680119 & Stuccato & $150000,0^{\circ}$ & $150003.7 \pm 0.53$ & $29 \pm 15$ & 39 & - \\
\hline 680126 & Cabriolet & 160000.11 & $160003.8 \pm 0.57$ & $33 \pm 16$ & 22 & - \\
\hline 680221 & Knax & $153000.0^{a}$ & $153001.9 \pm 0.52$ & $19 \pm 3.8$ & 162 & 5.8 \\
\hline 680229 & Dorsal Fin & 170830.04 & $170832.6 \pm 0.64$ & $25 \pm 6$ & 74 & $\mathbf{5 . 0}$ \\
\hline 680312 & Bugzy & 170400.11 & $170400.1 \pm 0.40$ & o & 19 & - \\
\hline 680322 & Stinger & $1500 \quad 00.04$ & $150.002 .1 \pm 0.50$ & $22 \pm 3.8$ & 136 & 5.6 \\
\hline 680325 & Milk Shake & 184427.04 & $184429.8 \pm 1.05$ & $26 \pm 11$ & 39 & - \\
\hline 680410 & Noor & $140000.0^{n}$ & $140002.8 \pm 0.63$ & $28 \pm 6.5$ & so & 4.6 \\
\hline 680418 & Shuffle & $140500.0^{2}$ & $140503.1 \pm 0.30$ & $35 \pm 3.9$ & 84 & 4.9 \\
\hline 680423 & Scroll & $170130.0^{n}$ & $170131.2 \pm 0.61$ & o & 29 & - \\
\hline 680426 & Boxcar & $150000.0^{2}$ & 150001.0 & $13 \pm 4$ & 261 & 6.2 \\
\hline
\end{tabular}


TABLE 1. (Continued.)

\begin{tabular}{|c|c|c|c|c|c|c|}
\hline \multirow[b]{2}{*}{ Date } & \multirow[b]{2}{*}{ Name } & \multicolumn{2}{|c|}{ Time, GMT (h-m-s) } & \multirow{2}{*}{$\begin{array}{c}\text { ISC } \\
\text { depth, km }\end{array}$} & \multirow{2}{*}{$\begin{array}{l}\text { No. obs. } \\
\text { to ISC }\end{array}$} & \multirow{2}{*}{$\begin{array}{l}\text { ISC } \\
m_{b}\end{array}$} \\
\hline & & Announced & ISC & & & \\
\hline 680517 & Clerksmobite & $130000.0^{n}$ & $130003.8 \pm 0.31$ & $36 \pm 5$ & 76 & 4.7 \\
\hline 680606 & Tub & $213000.0^{n}$ & $213002.6 \pm 0.83$ & $\mathbf{0}$ & 24 & - \\
\hline 680615 & Rickey & 135959.97 & $140001.4 \pm 15.7$ & $15 \pm 15.7$ & 180 & 5.9 \\
\hline 680628 & Chateaughy & $122200.0^{2}$ & $122201.2 \pm 0.45$ & $15 \pm 3.9$ & 142 & 5.3 \\
\hline 680730 & Tanya & $130000.0^{2}$ & $130001.0 \pm 0.44$ & 0 & 22 & - \\
\hline 680827 & Diana Moon & 163000.04 & $162959.9 \pm 0.30$ & $\mathbf{0}$ & 24 & - \\
\hline 680829 & Sled & $22 \quad 4500.04$ & $224500.7 \pm 0.80$ & $0 \pm 5$ & 192 & 5.9 \\
\hline 680906 & Knife $A$ & $1400 \quad 00.04$ & $135959.7 \pm 0.29$ & $\mathbf{0}$ & 42 & 5.8 \\
\hline 680906 & Noggin & $1400 \quad 00.13$ & $140002.0 \pm 0.63$ & $18 \pm 5.1$ & 109 & 5.5 \\
\hline 680917 & Stoddard & $1400 \quad 00.04$ & $140003.6 \pm 0.28$ & $36 \pm 3.5$ & 59 & 5.1 \\
\hline 680924 & Hudion Seal & $\begin{array}{lll}17 & 05 & 00.09\end{array}$ & $170503.3 \pm 1.47$ & $32 \pm 11$ & 40 & 5.0 \\
\hline 681003 & Knife C & 142900.04 & $142900 \pm 2.9$ & $5 \pm \mathbf{2 2}$ & 16 & - \\
\hline 681104 & Crew & 151500.09 & $151502.8 \pm 0.78$ & $27 \pm 6.7$ & 65 & 5.0 \\
\hline 681115 & Knife B & 154500.04 & $154500.8 \pm 0.63$ & 0 & 14 & - \\
\hline 681120 & Ming Vase & $\begin{array}{lll}18 & 00 & 00.03\end{array}$ & $1800 \quad 03.4 \pm 0.71$ & $30 \pm 6$ & $\mathbf{5 8}$ & 4.9 \\
\hline 681122 & Tinderbox & 161900,04 & $161900.3 \pm 0.29$ & o & 16 & - \\
\hline 681208 & Schooner & $1600 \quad 00.14$ & $160005.8 \pm 0.54$ & $42 \pm 8.4$ & 51 & 4.8 \\
\hline 681212 & Tyg & 151000.08 & $151003.0 \pm 0.27$ & $35 \pm 4$ & 43 & - \\
\hline 681219 & Berham & 163000.04 & $163002.0 \pm 0.50$ & $22 \pm 3.7$ & 240 & 6.3 \\
\hline 690115 & Packard & 190000.07 & $190005.2 \pm 0.48$ & $68 \pm 14$ & $\mathbf{2 5}$ & - \\
\hline 690115 & Wheskin & 193000.04 & $193002.1 \pm 0.57$ & $21 \pm 4.6$ & 101 & 5.3 \\
\hline 690130 & vise & 150000.04 & $1500 \quad 03.7 \pm 0.37$ & $32 \pm 4.8$ & 54 & 4.9 \\
\hline 690212 & Cypress & $\begin{array}{lll}16 & 18 & 20,88\end{array}$ & $161822.0 \pm 0.92$ & 17 & 33 & - \\
\hline 690320 & Bersec & $\begin{array}{lll}18 & 12 & 00.04\end{array}$ & $181203.5 \pm 0.68$ & $29 \pm 6$ & 35 & 4.4 \\
\hline 690321 & Coffer & $143000.0^{n}$ & $143002.9 \pm 0.77$ & $28 \pm 6$ & 53 & 4.9 \\
\hline 690430 & Blenton & $1700 \quad 00,04$ & $170002.6 \pm 0.67$ & $25 \pm 51$ & 105 & 5,2 \\
\hline 690430 & Thistle & $1700 \quad 00.04$ & $170002.6 \pm 0.67$ & $25 \pm 5.1$ & 105 & $\mathbf{3 . 2}$ \\
\hline 690507 & Purse & 134500.04 & $134501.9 \pm 0.59$ & $20 \pm 4.4$ & 163 & 5.5 \\
\hline 690527 & Torrido & 141500.04 & $141502.4 \pm 0.68$ & $24 \pm 5.9$ & 73 & $\mathbf{5 . 0}$ \\
\hline 690612 & Tapper & $1400 \quad 00.04$ & $140003.7 \pm 0.25$ & $36 \pm 4.4$ & 29 & 4.5 \\
\hline 690716 & Ildrim & 130230.04 & $130232.3 \pm 0.91$ & $21 \pm 8.7$ & 36 & 4.6 \\
\hline 690716 & Hutch & $1455 \quad 00.04$ & $145502.3 \pm 0.61$ & $22 \pm 5$ & 139 & 5.5 \\
\hline 690827 & Pliens & 134500.04 & $134503.0 \pm 1.1$ & 27 & 24 & - \\
\hline 690912 & Minute Strink & $\begin{array}{lll}18 & 02 & 20.42\end{array}$ & $180221 \pm 1,6$ & $10 \pm 12$ & 28 & - \\
\hline 690916 & Joram & 143000.04 & $143002.2 \pm 0.60$ & $2 \pm 4$ & 244 & 6.1 \\
\hline 691008 & Piplin & $14 \quad 30 \quad 00.14$ & $143001.7 \pm 9.53$ & $17 \pm 4$ & 145 & 5.6 \\
\hline 691029 & Cruet & 193000.04 & $193003.9 \pm 0.38$ & $32 \pm 17$ & 23 & - \\
\hline 691029 & Pod & $2000 \quad 00.04$ & $200002 \pm 1.0$ & $32 \pm 10$ & 33 & - \\
\hline 691029 & Calobuch & 220151.04 & $220152 \pm 1.0$ & $9 \pm 6.3$ & 145 & 5.6 \\
\hline 691121 & Plectini & $14 \$ 200.04$ & $145203.0 \pm 0.78$ & $27 \pm 6.4$ & 66 & 5.0 \\
\hline 691205 & Diesel Train & 170000.04 & $170002.6 \pm 0.74$ & $24 \pm 6.3$ & 65 & 4.9 \\
\hline 691217 & Gripe A & 150000.04 & $150002.5 \pm 0,60$ & $24 \pm 5.1$ & 112 & 5.4 \\
\hline 691117 & Lovage & 151500.04 & $151503.7 \pm 0.35$ & $21 \pm 5.3$ & 37 & 4.7 \\
\hline 691218 & Texrine & 190000.04 & $190003.7 \pm 0.32$ & $33 \pm 3.6$ & 85 & 5.2 \\
\hline
\end{tabular}


TABLE 1. (Continued.)

\begin{tabular}{|c|c|c|c|c|c|c|}
\hline \multirow[b]{2}{*}{ Date } & \multirow[b]{2}{*}{ Neme } & \multicolumn{2}{|c|}{ Time, GMT (h-m-t) } & \multirow{2}{*}{$\begin{array}{c}\text { S.SC } \\
\text { drpth, Im }\end{array}$} & \multirow{2}{*}{$\begin{array}{l}\text { No. obs. } \\
\text { to ISC }\end{array}$} & \multirow{2}{*}{$\begin{array}{l}\text { ISC } \\
m_{b}\end{array}$} \\
\hline & & Announced & ISC & & & \\
\hline 700123 & Fob & 163000.21 & $163000.4 \pm 0.34$ & $\mathbf{0}$ & 20 & - \\
\hline 700130 & Ajo & $1700 \quad 00.04$ & $170003.5 \pm 0.85$ & 31 & 32 & - \\
\hline 700204 & Grape B & 170000.04 & 17 of $01.8 \pm 0.52$ & $18 \pm 3.9$ & 152 & 5.6 \\
\hline 700203 & Labis & 150000.04 & $150002.0 \pm 0.80$ & 20 & 36 & 4.6 \\
\hline 700211 & Diane Mist & 191500.04 & $191502.1 \pm 0.74$ & $23 \pm 7.1$ & 41 & 4.7 \\
\hline 700225 & Cumarin & 142838.04 & $142840.8 \pm 0.78$ & $\mathbf{2 3}$ & 81 & 5.2 \\
\hline 700226 & Yannigan & 153000.04 & $153003.4 \pm 0.71$ & $28 \pm 5.7$ & $\mathbf{9 5}$ & 5.3 \\
\hline 700306 & Cyathus & 142400.94 & $142402.3 \pm 0.61$ & $25 \pm 5.8$ & 35 & 4.3 \\
\hline 700306 & Arabis & 150000.21 & $145959.7 \pm 0.23$ & 0 & 20 & - \\
\hline 700319 & Jali & $1403 \quad 30.04$ & $140329.9 \pm 0.19$ & $\mathbf{0}$ & $\mathbf{3 0}$ & - \\
\hline 700323 & Shaper & 230500.04 & $330502.1 \pm 0.62$ & $20 \pm 4.8$ & 139 & $\mathbf{5 . 5}$ \\
\hline 700326 & Handley & 190000.20 & $190002.4 \pm 0.47$ & $23 \pm 3.4$ & 260 & 6.4 \\
\hline 700421 & Snubber & $1430 \quad 00.04$ & $143003.1 \pm 0.84$ & $30 \pm 7.6$ & 39 & 4.4 \\
\hline 700421 & $\operatorname{Can}$ & 150000.04 & $150003.3 \pm 0.89$ & $30 \pm 7.6$ & 47 & 4.6 \\
\hline 700501 & Beebalm & 141300.04 & $141303.4 \pm 1.26$ & 32 & 29 & - \\
\hline 700501 & Hod & $14 \quad 40 \quad 00.17$ & $143959.2 \pm 0.29$ & $\mathbf{0}$ & 39 & 4.3 \\
\hline 700505 & Mint Leaf & $1530 \quad 00.17$ & $153001.8 \pm 0.67$ & $19 \pm 5.5$ & 77 & 5.0 \\
\hline 700515 & Comice & 133000.02 & $153001.0 \pm 1.000$ & 13 & 91 & 5.1 \\
\hline 700521 & Manzanir & $1400 \quad 00.04$ & $140003.3 \pm 3.7$ & 18 & 10 & - \\
\hline 700521 & Mocones & 141500.04 & $141503.5 \pm 0.30$ & $31 \pm 3.6$ & 81 & 5.1 \\
\hline 700526 & Hudson Moon & 141600.17 & $141602.8 \pm 1.1$ & 27 & 28 & - \\
\hline 700526 & Flask & 150000.05 & $150001.2 \pm 0.87$ & $14 \pm 5.5$ & 161 & 5.5 \\
\hline 700626 & Annice & 130000.04 & $130001.7 \pm 1.83$ & 13 & 27 & - \\
\hline 701014 & Tijerts & 143000.04 & $142959.5 \pm 0.77$ & $4 \pm 4.9$ & 195 & 5.5 \\
\hline 701105 & Abeytas & 150000.04 & $150002.7 \pm 0.87$ & 21 & 57 & 4.9 \\
\hline 701216 & Artesia & 160000.09 & $160001.9 \pm 1.12$ & $18 \pm 7.4$ & 39 & 5.2 \\
\hline 701216 & Cream . & 160000.17 & $160001.3 \pm 1.26$ & $18 \pm 7.8$ & $\mathbf{5 5}$ & 5.1 \\
\hline 701217 & Corpetbug & 160500.16 & $160501.3 \pm 0.77$ & $13 \pm 4.8$ & 173 & 5.8 \\
\hline 701218 & Banebetry & 153000.20 & $153001.9 \pm 0.67$ & $17 \pm 5.7$ & 76 & 5.1 \\
\hline 710616 & Emberdo & 145000.04 & $144959.0 \pm 0.51$ & $\mathbf{0}$ & 23 & - \\
\hline 710623 & Lepuna & 153000.04 & $153003.1 \pm 0.27$ & $35 \pm 4$ & 46 & - \\
\hline 710624 & Herrebell & 140000.16 & $140000 \pm 1.3$ & $6 \pm 9.6$ & 68 & 4.9 \\
\hline 710701 & Diemond Mine & 140000.14 & $135954.7 \pm 0.27$ & 0 & 7 & - \\
\hline 710708 & Mininen & 140000.08 & $135938.7 \pm 0.11$ & 0 & 160 & 5.5 \\
\hline 710818 & Algodone: & 140000.03 & $135959.3 \pm 0.84$ & $4 \pm 5.5$ & 130 & 5.3 \\
\hline 710929 & Pedernal & 140000.04 & $135958.7 \pm 0.37$ & $\mathbf{0}$ & 28 & - \\
\hline 711008 & Cethay & 143000.15 & $142958.8 \pm 0.39$ & $\mathbf{0}$ & 39 & - \\
\hline 711214 & Cheenacti: & 210959.16 & $210957.7 \pm 0.38$ & $\mathbf{0}$ & 43 & - \\
\hline 720419 & Longchemps & 163200.16 & $163158.7 \pm 0.49$ & $\mathbf{0}$ & 25 & - \\
\hline 720517 & Zinnia & 141000.16 & $140958.6 \pm 0.41$ & o & 31 & - \\
\hline 720519 & Monero & 170000.05 & $170000 \pm 2.2$ & $9 \pm 16$ & 50 & - \\
\hline $\mathbf{7 2 0 7 2 0}$ & Diamond Sculls & 171600.16 & $171502.9 \pm 0.68$ & $26 \pm 5.3$ & 76 & 4,9 \\
\hline 720921 & Oscuro & 153000.19 & $152958.9 \pm 0.09$ & a & 172 & 5.6 \\
\hline 720926 & Delphinium & 143000.15 & $142956.8 \pm 0.35$ & a & 37 & 4.1 \\
\hline
\end{tabular}


TABLE 1, (Continued.)

\begin{tabular}{|c|c|c|c|c|c|c|}
\hline \multirow[b]{2}{*}{ Duse } & \multirow[b]{2}{*}{ Name } & \multicolumn{2}{|c|}{ Time, GMT (h-m-s) } & \multirow{2}{*}{$\begin{array}{c}\text { ISC } \\
\text { depth, } \mathrm{km}\end{array}$} & \multirow{2}{*}{$\begin{array}{c}\text { No. obs, } \\
\text { to ISC }\end{array}$} & \multirow{2}{*}{$\begin{array}{c}\text { ISC } \\
\mathbf{m}_{b}\end{array}$} \\
\hline & & Announced & ISC & & & \\
\hline 721221 & Flax & $2015 \quad 00.24$ & $201458.7 \pm 0.18$ & $\mathbf{0}$ & 87 & 4.8 \\
\hline 730308 & Miera & 161000.19 & $160938.8 \pm 0.11$ & $\mathbf{0}$ & 161 & 5.3 \\
\hline 730425 & Angus & 222500.03 & $222502.2 \pm 0.98$ & $29 \pm 9.5$ & 48 & 4.5 \\
\hline 730426 & Starwort & 171500,16 & $171459.2 \pm 0.72$ & $1 \pm 4.5$ & 168 & 5.6 \\
\hline 730605 & Dido Queen & $1700 \quad 00.17$ & $165959.0 \pm 0.18$ & $\mathbf{0}$ & $\mathbf{B 2}$ & 5.0 \\
\hline 730606 & Alinendro & $130000 . c 8$ & $130000.0 \pm 0.60$ & $7 \pm 3.7$ & 258 & 6.1 \\
\hline 730628 & Portuleca & 191512.40 & $191511.2 \pm 0.19$ & $\mathbf{0}$ & 91 & 4.9 \\
\hline 731012 & Husky Ace & $\begin{array}{lll}17 & 00 & 00.08\end{array}$ & $165959.1 \pm 0.19$ & $\mathbf{0}$ & $\mathbf{5 7}$ & 4.7 \\
\hline
\end{tabular}

Acrual detonation time was about $0.1 \pm 0.06 \mathrm{~s}$ later.

TABLE 2. Announced and ISC data for U.S. Tests on Amchitka, from Springer and Kinaaman. The ISC origin time for Milrow is based on a calculated depth of $34 \pm \mathrm{km}$. The ISC origin time for Longshot is based on an assumed depth of $0 \mathrm{~km}$ and for Cannikin on a depth of $2 \mathrm{~km}$. These assumed depths are good approxinations to the actual depths of 1.2 and $1.8 \mathrm{~km}$, respectively.

\begin{tabular}{|c|c|c|c|c|c|}
\hline \multirow[b]{2}{*}{ Date } & \multirow[b]{2}{*}{ Name } & \multicolumn{2}{|c|}{ Time, GMT (h-m-s) } & \multirow{2}{*}{$\begin{array}{l}\text { No. obs. } \\
\text { to ISC }\end{array}$} & \multirow[b]{2}{*}{ ISC $m_{b}$} \\
\hline & & Announced & ISC & & \\
\hline 651029 & Longthot & $2100 \quad 00.08$ & $\begin{array}{llll}21 & 00 & 03.6 & \pm 0.08\end{array}$ & 233 & 5.8 \\
\hline 691002 & Milrow & 22 of 00.04 & $220601.9 \pm 0.75$ & 294 & 6.4 \\
\hline 711106 & Cennikin & 220000.06 & $215956.8 \pm 0.06$ & 374 & 6.6 \\
\hline
\end{tabular}

TABLE 3. Announced and ISC data for French Tests in the Sahara, from Duclaux and Michaud. The ISC assumed a depth of $0 \mathrm{~km}$ for all these events. The 1SC did not publish any datn for Agate on 611107, Emeraude on 630318, Amethyst on 630330, Opal on 640214, Topaze on 640615, Turquoise on 641128, Jade on 650330, and Corindon on 651001. The Sahara tests were conducted in a granite massif of the Hoggar, the Taourirt Tan Afela, which is located at approximately $24^{\circ} \mathrm{N}-5^{\circ} \mathrm{E}$.

\begin{tabular}{|c|c|c|c|c|c|}
\hline \multirow[b]{2}{*}{ Dese } & \multirow[b]{2}{*}{ Name } & \multicolumn{2}{|c|}{ Time, GMT (h-m-t) } & \multirow{2}{*}{$\begin{array}{c}\text { No. obs. } \\
\text { to ISC }\end{array}$} & \multirow[b]{2}{*}{ ISC $\mathbf{m}_{b}$} \\
\hline & & Announced & ISC & & \\
\hline 620501 & Beryl & $1000 \quad 00.458$ & 095959 & 73 & - \\
\hline 631029 & Rubis & 130000.011 & 125959 & 124 & - \\
\hline 650227 & Saphir & 113000.039 & $112958.6 \pm 0.12$ & 132 & 5.6 \\
\hline 651201 & Tourmaline & 103000.088 & $102958.4 \pm 0.24$ & 49 & 4.9 \\
\hline 660216 & Grenat & 110000.035 & $110000^{2}$ & 57 & - \\
\hline
\end{tabular}

Time given by Rabar (RBA), Morocco. 
TABLE 4. ISC data for presumed Soviet tests at the Nuclear Testing Ground (NTC) in Eastern Kazakhstan. The ISC assumed a depth of $33 \mathrm{~km}$ for the events of 661203 and 710630 , and a depth of $0 \mathrm{~km}$ for all other events. The locetions of these tests extend from approximately 78 to $79^{\circ} \mathrm{E}$ at $50^{\circ} \mathrm{N}$.

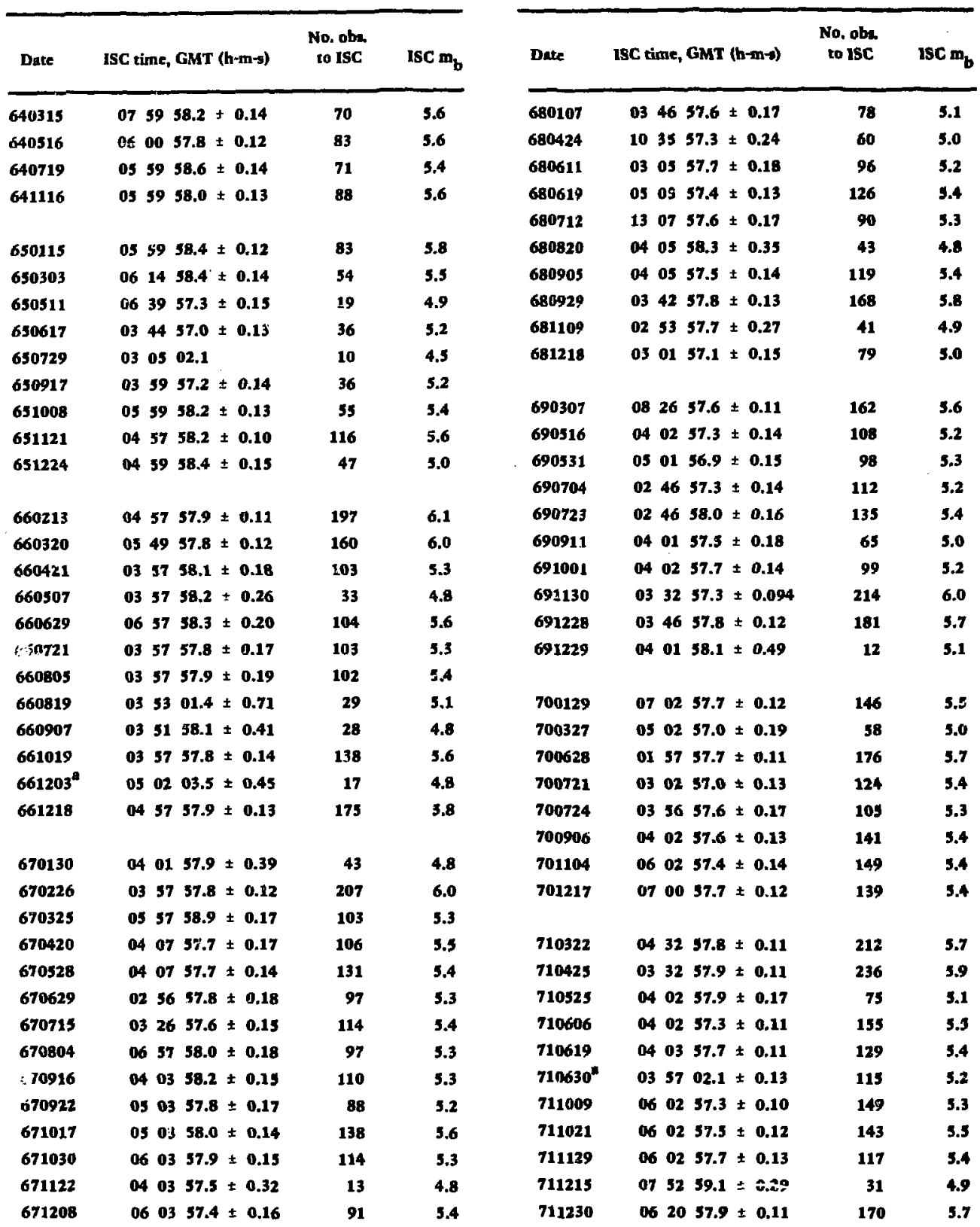


TABLE 4. (Continued,)

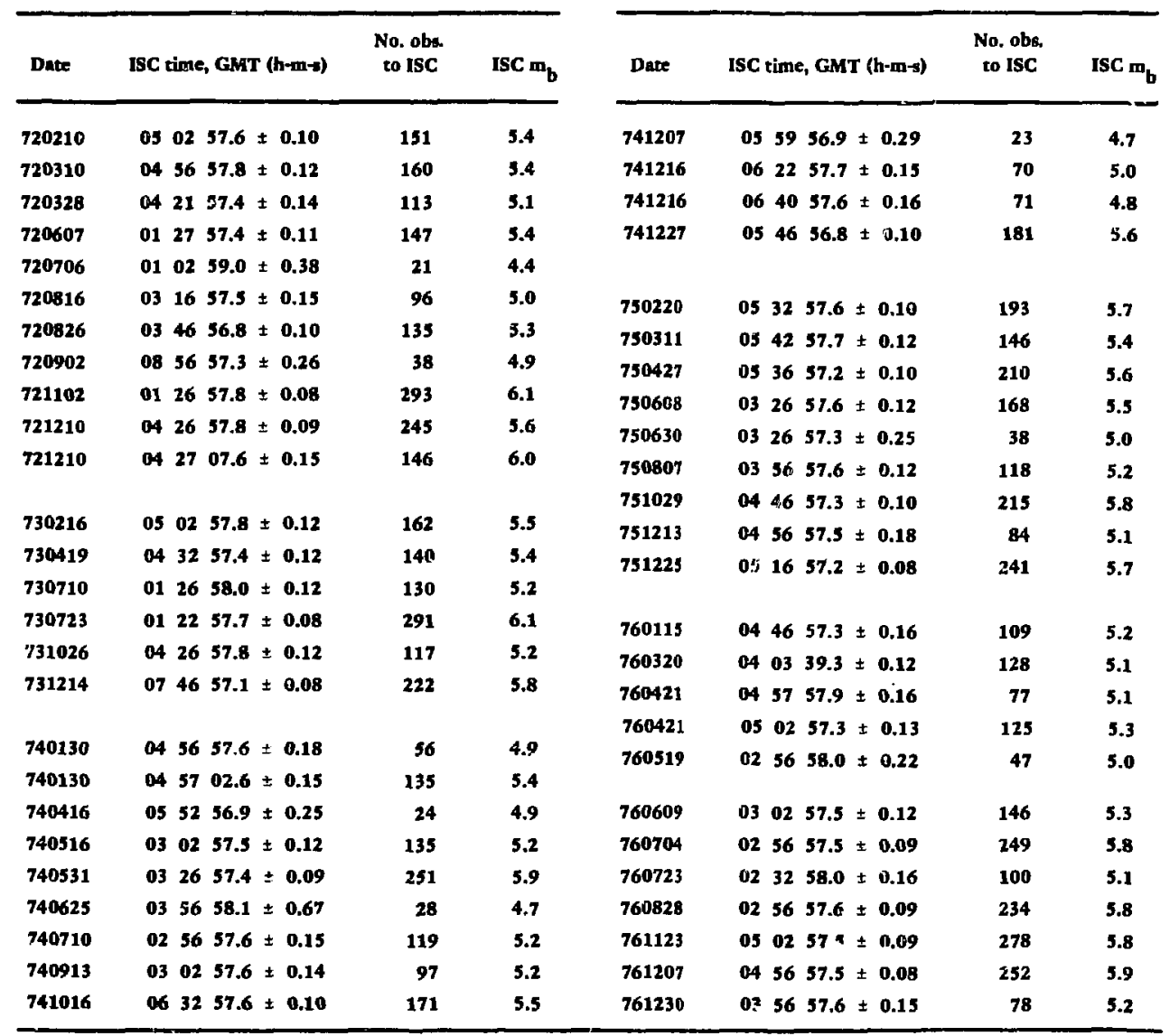

ASC depth $=33 \mathrm{~km}$. 
TABLE 5. ISC data for presumed Soviet tests on Novaya Zemlya. A depth of $0 \mathrm{~km}$ was assumed by the ISC for all these events.

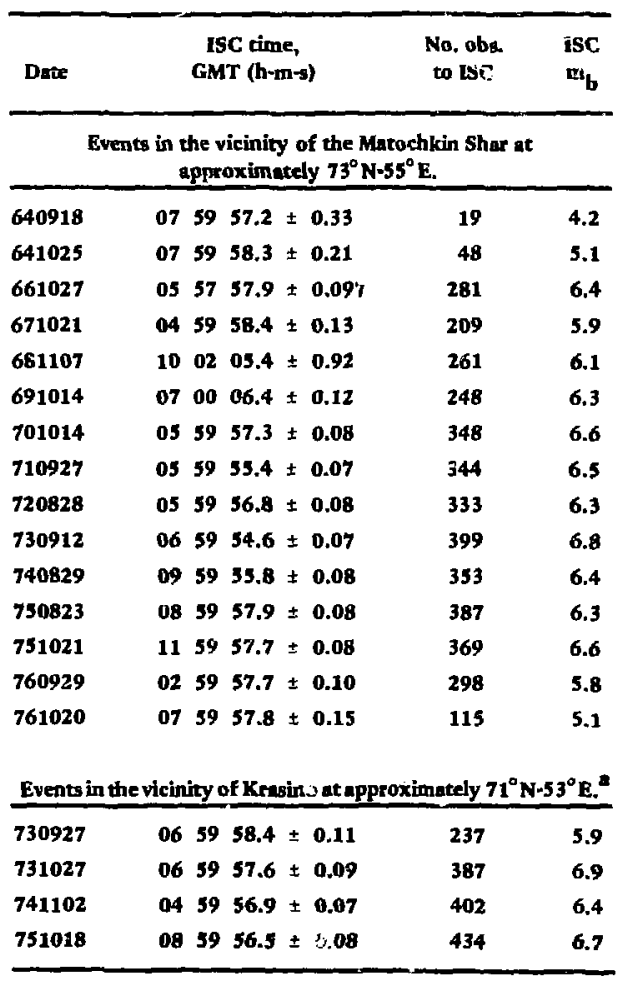

Two probable aftershocks of the event on 731007, which oceurred on 740707 and 740722 , are not included.
TABLE 6. ISC data for presumed Soviet tests near Azgir at approximately $48^{\circ} \mathrm{N}-48^{\circ} \mathrm{E}$. A depth of $33 \mathrm{~km}$ was assumed for the event of 660422 ; ir was not listed as a probable explosion. A depth of $29 \pm 1.7 \mathrm{~km}$ was calculated for the event of 680701 . A depth of $0 \mathrm{~km}$ was assumed for the other three events.

\begin{tabular}{lcccc}
\hline Date & ISC time, GMT (h-m-s) & $\begin{array}{c}\text { No. oba. } \\
\text { to ISC }\end{array}$ & ISC mb \\
\hline 660422 & 0258 & $04.0 \pm 0.37$ & 38 & 4.7 \\
680701 & $040200.9 \pm 0.17$ & 166 & 5.5 \\
711222 & $065956.5 \pm 0.13$ & 230 & 6.0 \\
750425 & $050003.0 \pm 1.8$ & 24 & 4.7 \\
760729 & $045958.0 \pm 0.09$ & 297 & 5.9 \\
\hline
\end{tabular}

TABLE 7. ISC data for presumed Soviet tests on the Ustyurt Plateau at approximately $44^{\circ} \mathrm{N}-55^{\circ} \mathrm{E}$. The ISC assumed a depth of $0 \mathrm{~km}$ for these events.

\begin{tabular}{|c|c|c|c|}
\hline Dute & ISC tine, GMT (h-m-t) & $\begin{array}{c}\text { No. obs. } \\
\text { to ISC }\end{array}$ & $1 S C \mathbf{m}_{\mathbf{b}}$ \\
\hline 691206 & $0702 \quad 57.5 \pm 0.093$ & 180 & 5.8 \\
\hline 701212 & $070057.4 \pm 0.09$ & 246 & 6.0 \\
\hline 701223 & $070057.3 \pm 0.92$ & 254 & 6.0 \\
\hline
\end{tabular}

\section{ANAI,YSIS OF ORIGIN TIMES}

\section{NTS, NEVADA}

The announced detonation times (after the minute) for the 217 NTS explosions listed in Table 1 are plotted in Fig. 1. Most of the detonations occurred within the first second after the minute, a few during the thirtieth second, and a very few at other times during a minute. Of the 181 detonations occurring within the first second, 179 were in the interval from 0.0 to $0.2 \mathrm{~s}$.

With a few exceptions, the ISC origin times for events at the other test sites are based on teleseismic observations at distances greater than $20^{\circ}$. This is in contrast to the ISC origin times for the NTS explosions that are based on boch near-regional and regional $\left(0.1\right.$ to $\left.20^{\circ}\right)$ and teleseismic $\left(>20^{\circ}\right)$ distances. The only observations for many of the lower-magnitude explosions were at regional distances. The use of regional as well as teleseismic observations is probably responsible for some of the differences in the distributions of origin times for events at the NTS and events at the NTG and Novaya Zemlya (non-Gaussian vs approximately Gaussian). 


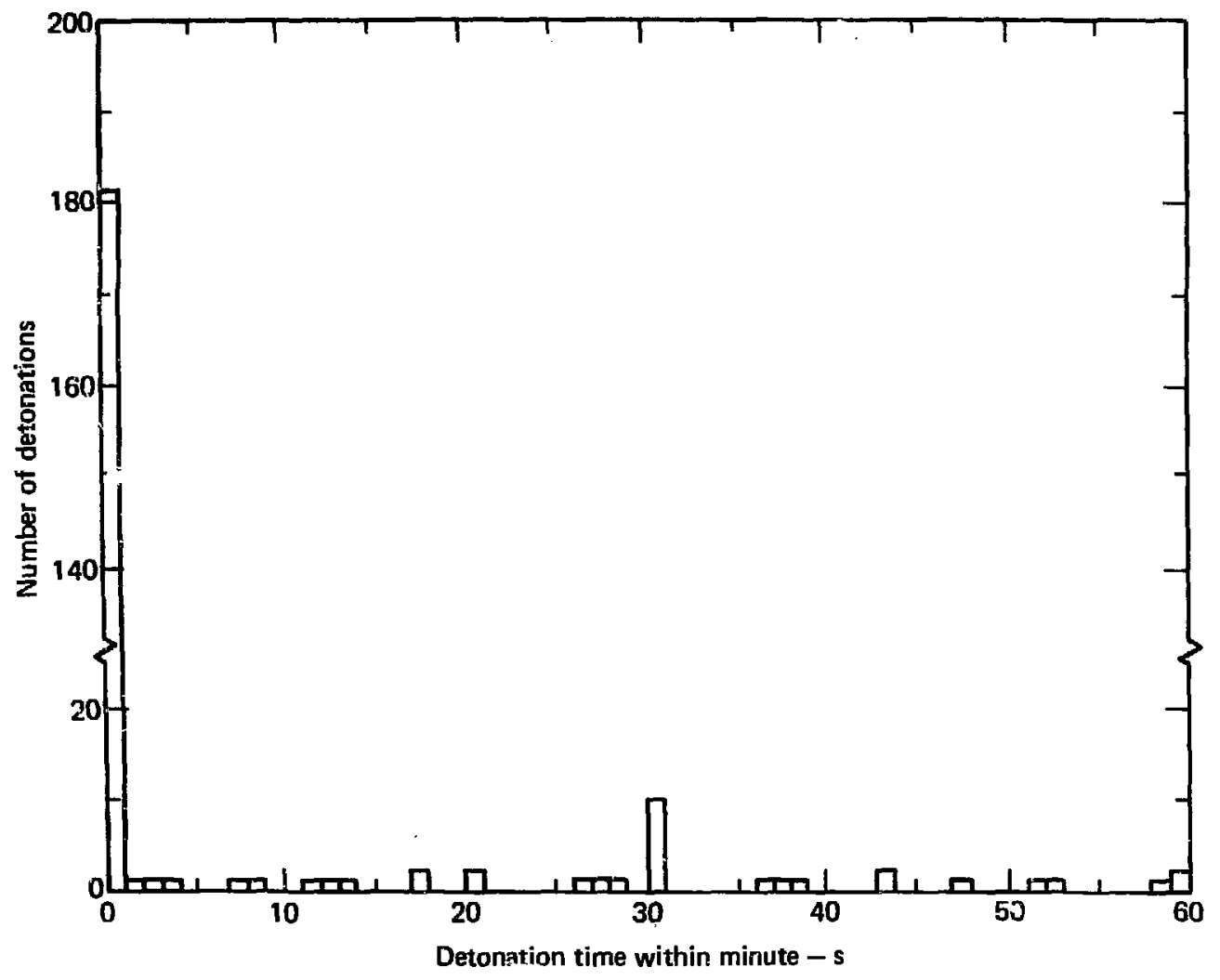

FIG. 1. Incremental distribution of detonation times within (8fter) the minute for the 217 NTS explosions listed in Table 1.0 f the 181 deto iations during the finst second, $175 \mathrm{w}$ gre in the interval from 0.0 to $0.2 \mathrm{~s}$. The distributions of ISC origin times within the mingte for iwo subsets and th. full set of these 175 explesions are presented in Figs. 2 through 4.

The ISC used different practices to calculate origin times and epicentral locations for events at NTS vs events at the other test sites. The ISC (with a few exceptions listed in Tables 2,4 , and 6 ) assumed zero depth in the calculations for events at sites other than NTS. The ISC calculated an event depth at the NTS whenever the data permitted (generally higher-magnitude events with many teleseismic as well as regional observations). The ISC calculated nonzero depths for 123 of the 175 NTS explosions whose origin times are a subject of this study. The ISC calculated a zero depth for one explosion and assumed zero depths for the remaining 51 of the 175 explosions. These calculated nonzero depths are plotted vs the calculated origin times in Fig. 2. The
ISC uses thi Jeffrey-Bullen tables in calculating origin time, epicentral location, and depth; the Pwave velocity at a depth of $33 \mathrm{~km}$ in these tables is $7.76 \mathrm{~km} / \mathrm{s}$ (Bullen, 1963, p. 223). It appeared reasonable from Fig. 2 to use this velocity to extrapolate the origin times of the 123 events shown in Fig. 2 to zero-depth values; this was done with the result shown in the top of Fig. 3. The ISC origin times for the 52 zero-depth NTS explosions are plotted in the bottom of Fig. 3. Note that the mean origin time for the zero-depth explosions is somewhat later than the mean time for the other 123 explosions extrapolated to zero depth. The combined incremental distribution of origin times for the 175 NTS explosions is presented in the middle 


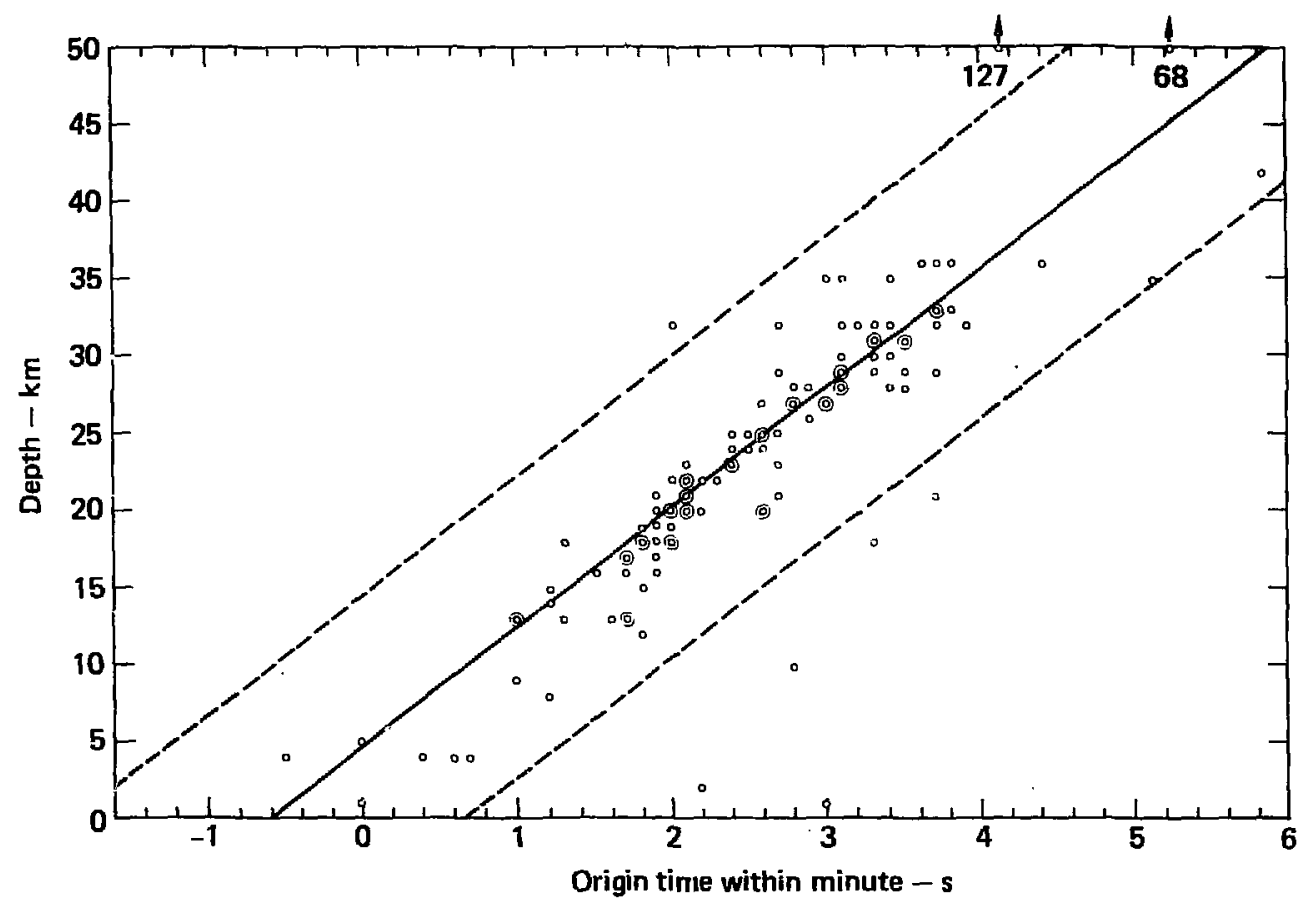

FIG. 2. ISC depth vs ISC origin times within (before and after) the minute for 123 of the 217 NTS explosions listed in Table 1. These 123 events are a subset of the 175 explosions with detonation times in the interval from 0.0 to $0.2 \mathrm{~s}$ after the minute. The ISC calculated nonzero depth for these events. The mean variation of ISC depth vs ISC origin time, and the correspending standard deviation about the mean, are plotted, assuming a seismic velocity of $7.76 \mathrm{~km} / \mathrm{s}$.

of Fig. 3. The cumulation distribution of the origin times for the 175 NTS explosions is presented in Fig. 4, together with the calculated mean and standard deviation of the origin times for these explosions. As shown below, the distribution of the origin times for the 175 NTS explosions does not approximate a Gaussian distribution as well as do the distributions for events at NTG and Novaya Zemlya.

\section{NTG, EASTERN KAZAKHSTAN}

The ISC origin times before and after the minute for the 129 NTG events listed in Table 4 are plotted to the nearest second in Fig. 5. The times for 122 events are concentrated in the interval 1 to $3 \mathrm{~s}$ before the minute. The origin times of the other seven of the 129 events have plausible explanations.
For example, the origin times of the events of 721210 and 740130 are very easy to explain. There were double events at NTG on each of these days. Those on $\mathbf{7 2 1 2 1 0}$ were separated by approximately $10 \mathrm{~s}$ and those on 740130 by approximately $5 \mathrm{~s}$. According to the ISC, the first events on these days occurred 1 to $3 \mathrm{~s}$ before the minute, suggesting that the first explosion of each pair was detonated according to the standard timing practice at NTG.

The ISC assumed depths of $33 \mathrm{~km}$ for the events of 661203 and 710630 and depths of $0 \mathrm{~km}$ for the other 127 NTG events. As a consequence, the apparent travel times for the events of 651203 and 710630 were approximately $4 \mathrm{~s}$ shorter than for the events assumed to be at zero depth. If the travel times for these two events were increased by approximately this amount, their apparent origin times would be from $I$ to $3 \mathrm{~s}$ before the minute, as is the case for 122 of the 129 NTG events. 

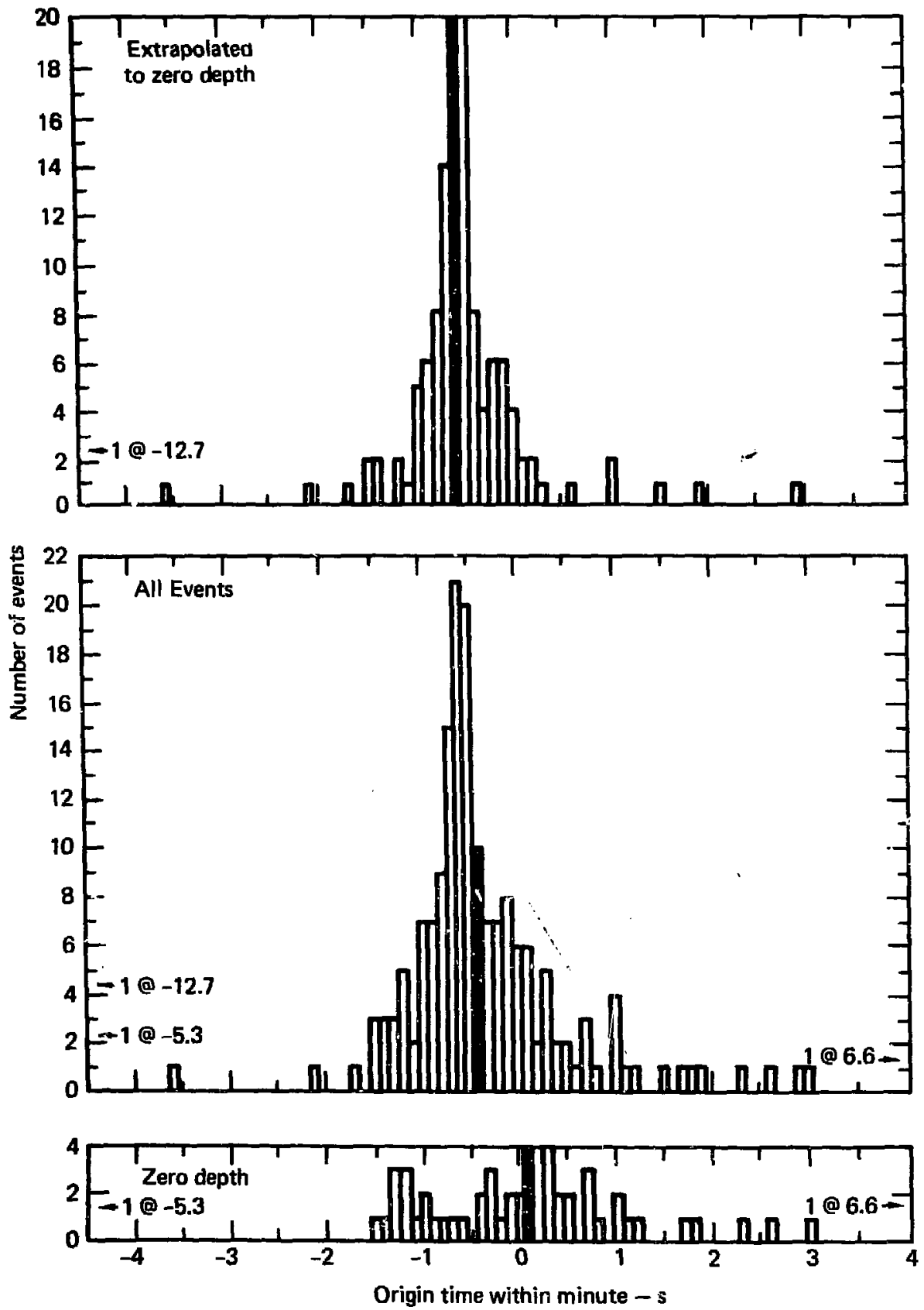

FIG. 3. Incremental distribution of origin times within (before and after) the minute for the 175 NTS explosions listed in Table I, with detontion times in the interval from 0.0 to $0.2 \mathrm{~s}$ after the minute. In the top part of the Figure, the origin times of the 123 evenics of Fig. 2 are extrapolated to zero depth, assuming a seismic velocity of $7.76 \mathrm{~km} / \mathrm{s}$. The origin times for the 52 events with zero ISC depth are presented in the lower part of the figure. The ortein time distribution, ascuming zero depths for all 175 events, is given in the middle part of the figure. The Filled-in bars indicate the mean origin time for each subset and the full set of the 175 events. 
The events of 650729 and 660819 were detected and reported by 10 and 28 stations, respectively, in Europe and North America. An additional station in Trinidad in the Caribbean region reported diffracted $P$ from the event of 660819 . The azimuth ranges for these reporting stations relative to the NTG are only 297 to $005^{\circ}$ (for 650729) and 291 to $021^{\circ}$ (for 660819). As demonstrated by Dahlman and Israelson (pp 176-180) in a calculational experiment involving 19 explosions with known locations at NTS, $90 \%$ of the random location errors were less than $57 \mathrm{~km}$ with data from 8 to 10 stations located within an azimuth section of $90^{\circ}$. For 8 to 10 stations well distributed in azimuth, $90 \%$ of the random location errors were less than $12 \mathrm{~km}$. The ISC located these two events about 60 to $70 \mathrm{~km}$ northnorthwest of the central concentration of most
NTG event locations. A shift of these two events to locations within this central concentration would increase the apparent travel times to the stations in Europe and North America and shift the apparent origin times toward the range from 1 to $3 \mathrm{~s}$ berore the minute.

The origin time for the event on 760320 is slearly anomalous, as shown in Fig. 5. If that event was an explosion, there was a clean departure in that case from what appears, according to Fig. 5, to be standard Soviet nuclear testing practice. However, the event of 760320 would not be unusual in the context of U.S. nuclear testing practice as shown in Fig. I. The event of 760320 couid be an earthquake, a clearly unusual event for that aseismic area. Either explanation is plausible. Whatever the true nature of that event, its origin

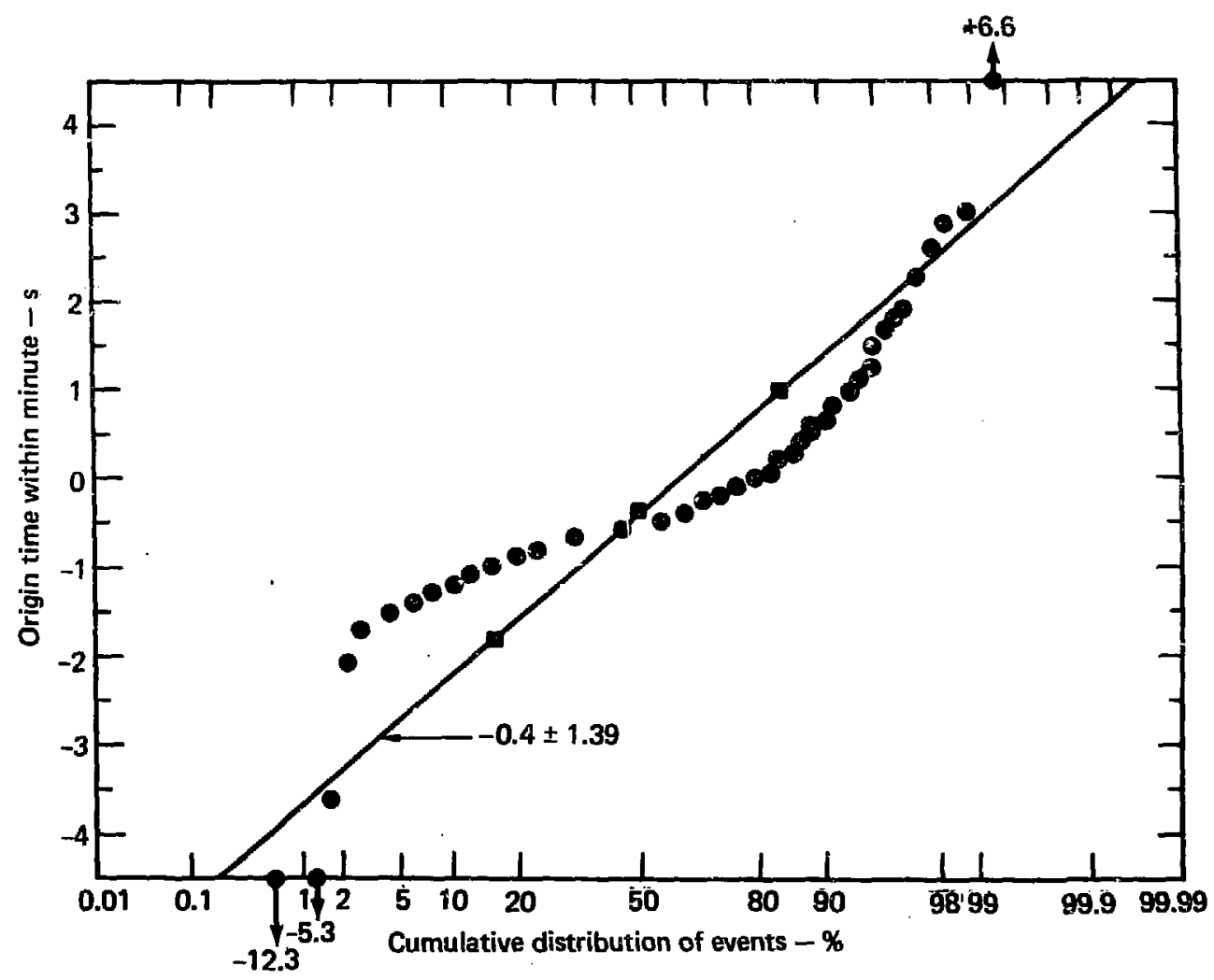

F1G. 4. Cumniative distribution of the origin times for the 175 events plotted in the middle part of Fig. 3. The ideal normal distribution with the same mean and standard devintion is also plotted. It is dear that the origin-time distribution is not Gaussian. 


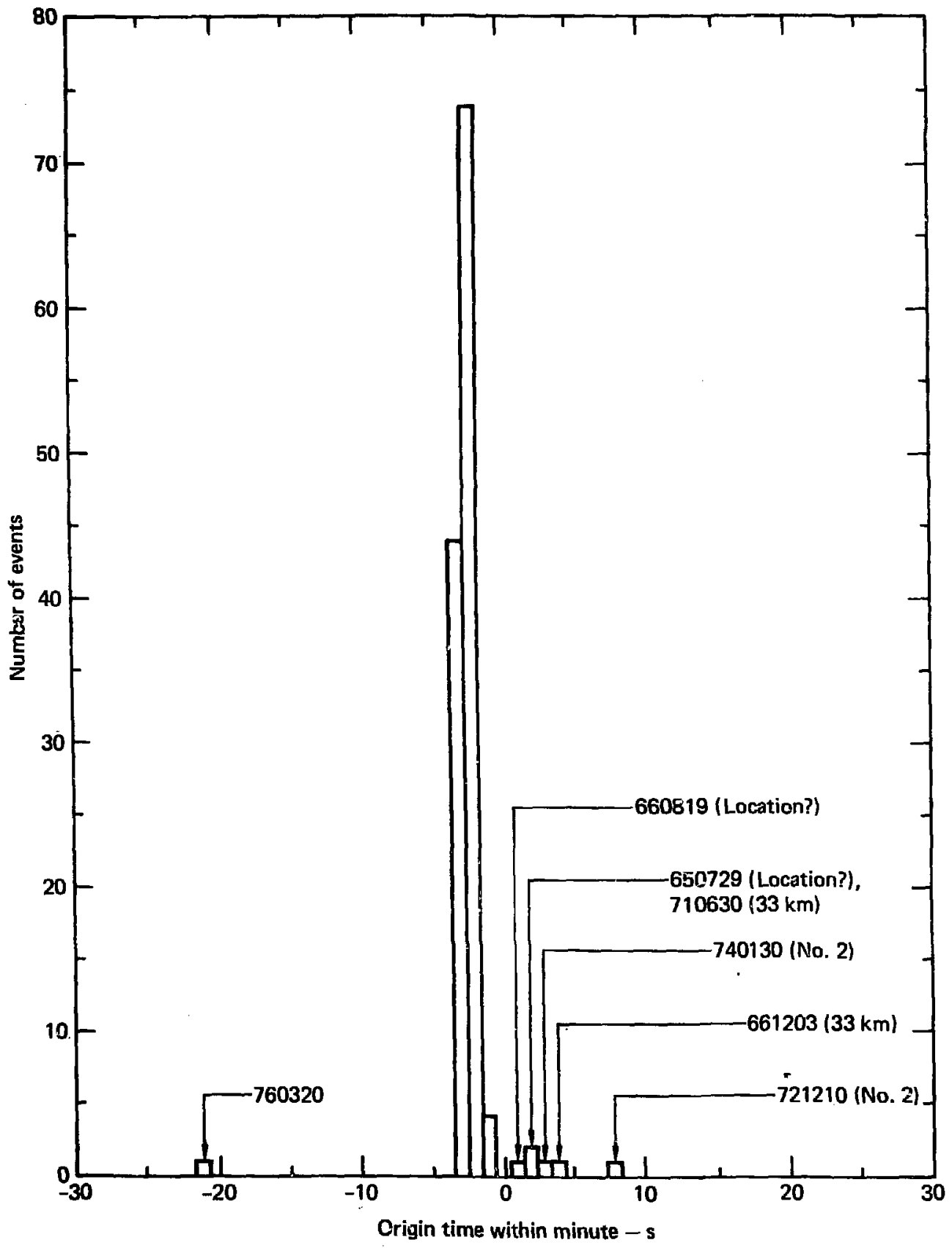

F1G. 5. Incremental distribution of ISC origin times within (before and after) the minute for the 129 NTG events listed in Table 4. The outliers among the events are identified by date. The events on 650729 and 660819 were probably mislocated by the ISC. The ISC set the depths of the events on 661203 and 710630 at $33 \mathrm{~km}$. The symbal No. 2 means that the event of 721210 followed another event by approximately $10 \mathrm{~s}$, and that the event of 740130 followed another event by approximately $5 \mathrm{~s}$. The event of 760320 was either a rare earthquake in this aseismic area or an explosion that was not detomated according to standard timing practice. 
time does not fit within the statistical pattern of those for the 122 NTG events that are not identified by date in Fig. 5 .

In summary, there are plausible explanations why the ISC origin times for the seven events identified by date in Fig. 5 are not in the interval from 1 to $3 \mathrm{~s}$ before the minute, which is the case for 122 of the 129 NTG events. The detonation times for four of the seven events could have been essentially the same-within the minute-as those for the 122 NTG events. The other two of the seven events were deliberately detonated approximately 5 and $10 \mathrm{~s}$ later than the standard time. The origin time of the seventh event, that of 760320 , does not fit the pattern for the other 128 events.

In the following, only the 122 events with origin times in the range from $I$ to $3 \mathrm{~s}$ before the minute are considered. The incremental origin time distribution for these events is shown in Fig. 6 and the cumulative distribution in Fig. 7. The calculated mean and standard deviations for these events are also given in Fig. 7. The distribution of the ISC origin times for these events is a much betier approximation to a Gaussian distribution than that for NTS explosions (compare Fig. 4). The standard deviation of the origin time for NTG events is much smaller than that for NTS events. It is also clear from Figs. 3, 4, 6, and 7 that the mean NTG event origin time is almost two seconds earlier than that for NTS explosions.

\section{OTHER TEST SITES}

The ISC origin times from Tables 2, 3, and 5-7 -for the Amchitka, Sahara, Azgir, Ustyurt, and Novaya Zemlya test sites are plotted in Fig. 8. As indicated, the origin times for one event on Amchitka and two near Azgir are extrapolated to zero depth. The times for Amchitka and Azgir are rather scattered. They are less so for the Sahara, but as indicated in Tiable 3, the origin times appear to have been determined in three differ'nt ways. The data for Ustyurt are very consistent. Those for Novaya Zemlya are reasonably so, except for two events on 681107 and 691014 , with origin times 5.4 and $6.4 \mathrm{~s}$ after the minute, respectively. Inspection of Table 5 indicated that the daia for the two sites on Novaya Zemlya could be considered together. The distribution of the origin times for Novaya Zemlya, except for the two after the minute, is a fair upproximation to a Gaussian distribution as shown in Fig. 9.

\section{ORIGIN TIMES AND $P_{n}$ VELOCITIES}

The origin times for the U.S. and French explosions plotted in Figs. 2-4 and 8 are for explosions with announced detonation times in the interval from 0.0 to $0.2 \mathrm{~s}$ after the minute. It is reasonable to assume that the detonation times corresponding to the origin times for the NTG explosions plotted in Figs. 6 and 7 are also in this interval. The statistics are much poorer for the other three Soviet test sites, but it is appropriate to assume similar detonation times for the events shown in Fig. 8 for Azgir, Ustyurt, and Nova Zemlya-except for the events on 681107 and 691014 at the latter site.

In Table 8, the average origin time (before the minute) for the seven test sites are presented together with $P_{n}$ velocities beneath those sites. No $P_{n}$ velocity data are available for Novaya Zemlya, but extrapolation from the data for the Urals in Volvovskii (1973) suggests a value of $8.2 \mathrm{~km} / \mathrm{s}$ or greater. There is a fairly consistent trend in Table 8 of earlier origin times with higher $P_{n}$ velocities. This is consistent with the working hypothesis of Marshall, et al. that a low $P_{n}$ velocity indicates low upper-mantle velocities, and a high $P_{n}$ velocity indicates high upper-mantle velocities. 


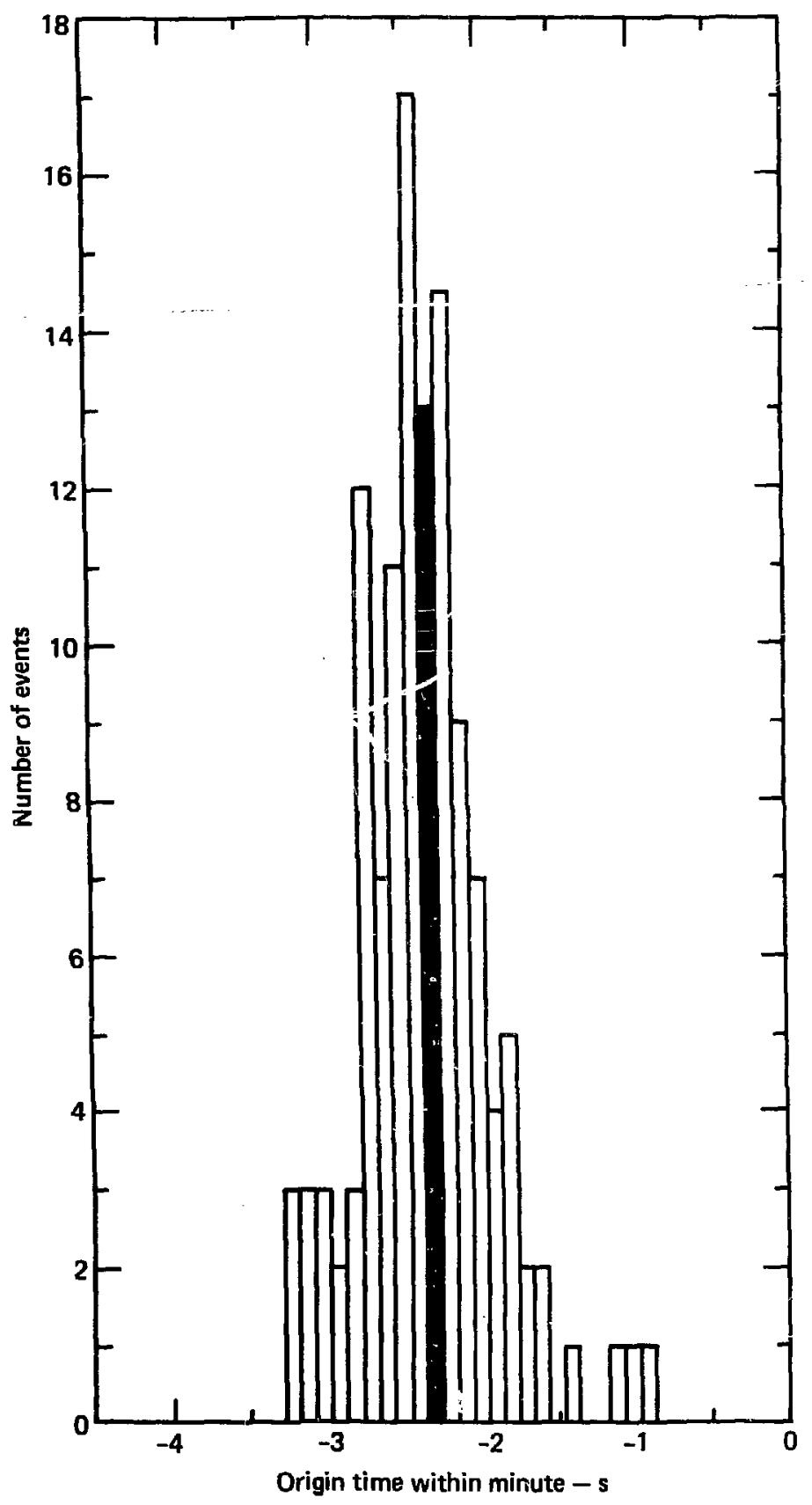

FIC. 6. Incremental distribation of ISC origin time within (before and after) the minute for 122 of the 129 NTG events listed in Table 4. The seven events identifled by date in Fig. 5 are not included. The filled-in bar indicates the menn origin time for these 122 events. 


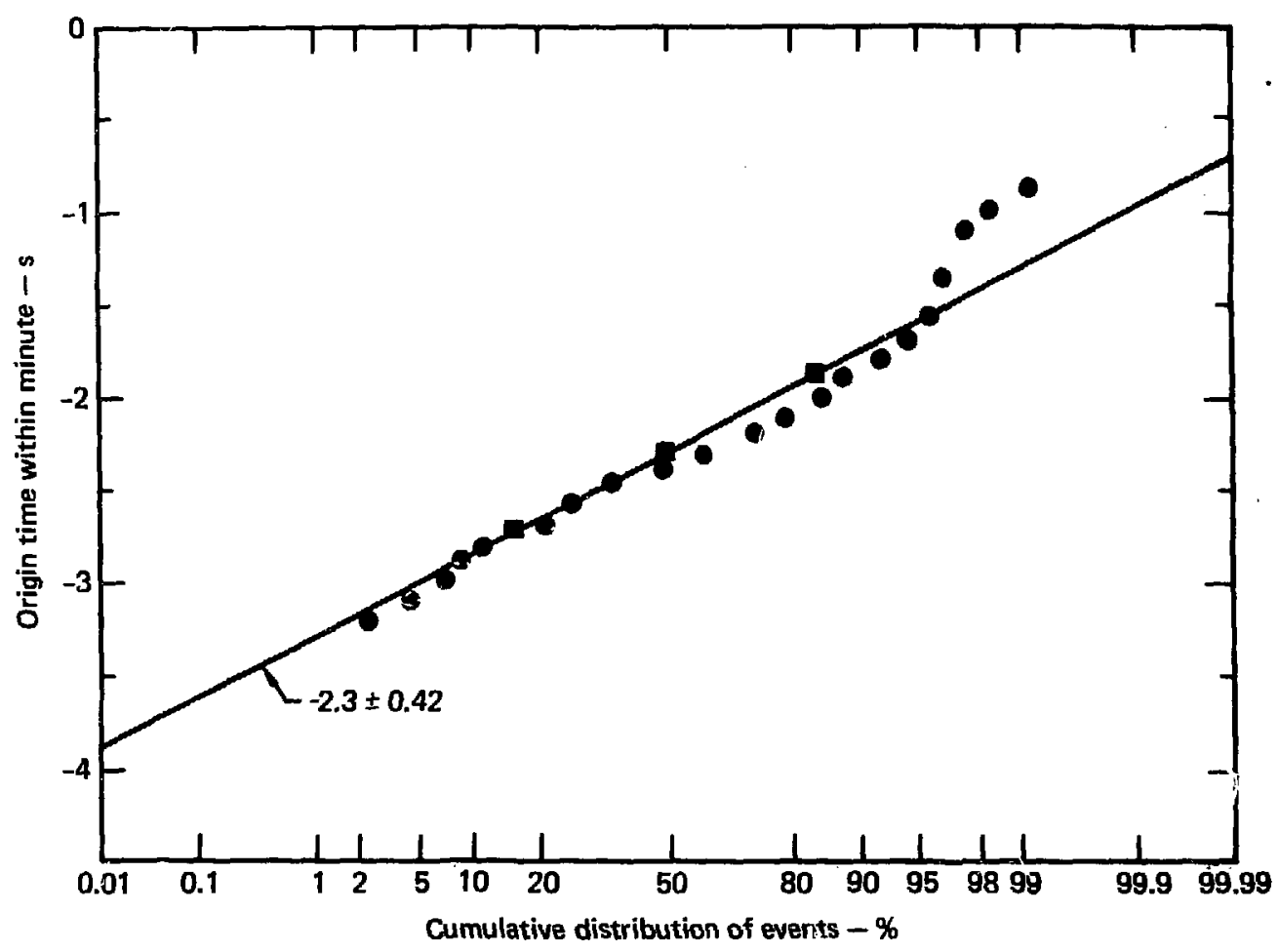

FIG. 7. Cumulative distribution of ISC origin times within (before) the ininute for the 122 NTG events of Fig. 6. The ideal normal distributlon with the same mean and standard deviaticn is also plotted. It is clear that the distribution of the NTG erighs time is a much better approximation to tue Gaussian than the NTS distribution in Fig. 4. 

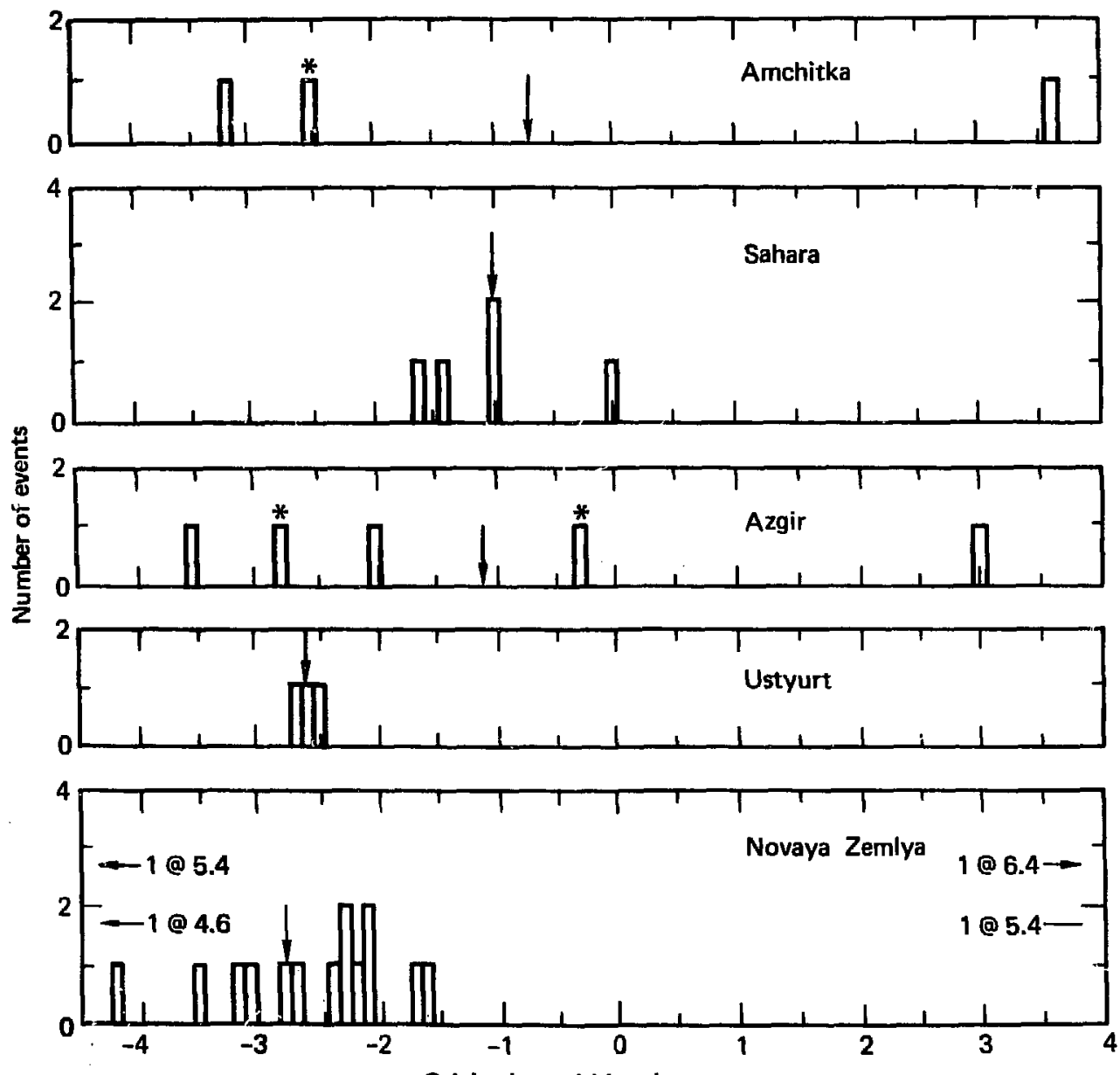

$\frac{1}{4}$

Origin time within minute $-\mathrm{s}$

FIG. 8. Incremental distribution of ISC origin times within (before and after) the minute for events on Amchilka, in the Suhara, mear A zoghr, on the Ustyurt Plateau, and on Novaya Zemlya. The asterisks indicate that the origin time was extrapolated to zero depth, assuming a seistnic velocity of $7.76 \mathrm{~km} / \mathrm{s}$. The arrows indicate the mean origin times. The events on Novaya Zemlya with origin times of 5.4 and $6.4 \mathrm{~s}$ (on 681107 and 691014 ) were not ured in calculating the mean origin time. The data are from Tables $2,3,5,6$, and 7. 


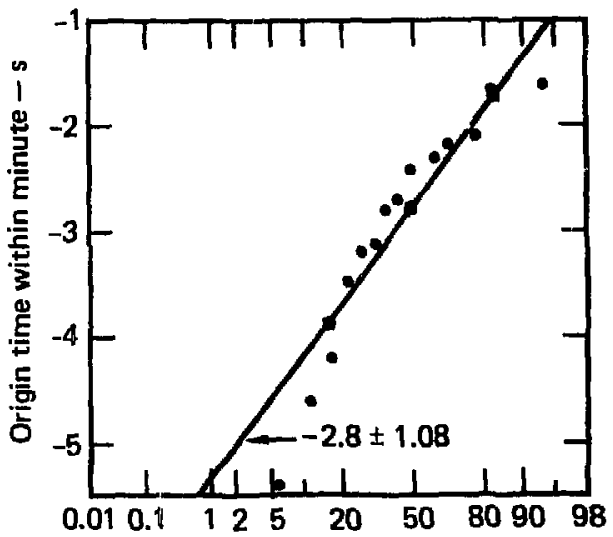

Cumulative distribution of events $-\%$

FIG. 9. Cumulative distribution of ISC origln times within (before) the minute for 17 of the Novaya Zemlya events listed in Table 5 . The events on 681107 and 691014 are not included. The ideal normal distribution with the same mean and standard deviation is also plotted. The origin time distribution is a fair approximation to the Gaussian, better than that for NTS but not as good as for the NTG.
TABLE 8. Average ISC origin times within (before) the minute and $P_{n}$ velocities for the sereral testing oites. The average origin times are from Figs. 3,6, and 8. The $\mathbf{P}_{\mathbf{n}}$ velocities are those given by Marai iall, et, all, except that for the Ustyurt Platean which is from Yolvovgkil.

\begin{tabular}{|c|c|c|}
\hline Test xite & Menn origin timc, & velocity, $\mathrm{km} / \mathrm{s}$ \\
\hline NTS & -0.4 & 7.8 \\
\hline Sahore & -1.0 & 7.84 \\
\hline Amchitie: & -0.7 & 6,05 \\
\hline Axghr & -1.1 & 8.15 \\
\hline NTG & $-2,3$ & 8.3 \\
\hline Vatyurt & $-2.6 j$ & 8.4 \\
\hline Novaya zemlya & $-7 / 8$ & Not availnble \\
\hline
\end{tabular}

\section{CONCLUSIONS}

The detonation times for U.S. and French underground nuclear explosions are generally within one or two tenths of a second after the minute. The seismic evidence indicates that the normal Soviet practice is very similar.

The statistics for the origin times of NTS explosions could be improved by using the same procedure used by the ISC for calculating the origin times for NTG events: assume zero depth and use only teleseismic observations.

The statistics of the calculated origin times for all test sites would be improved if the origin times were calculated in accord with one or more of the following:

- Use only teleseismic observations.

- Use announced locations of U.S. and French explosions.

- Use the announced altitude above or depth below sea level for U.S. explosions; assume zero depth at the altitude of the test site for the other explosions.

- Weight the calculated origin time for each event according to its estimated standard error.

The mean origin time for NTG events is significantly earlier (approximately two seconds) than that for NTS events. Assuming comparable detonation timing, this difference indicates significantly higher seismic velocities in the crust and upper mantle beneath NTG as compered to NTS. Similarly, the seismic velocities beneath Amchitka and the Sahara appear to be less than the velocities beneath the Azgir, Ustyurt, and Novaya Zemlya sites.

The mean origin time data for NTS, NTG and the other test sites are consistent with a direct correlation between $P_{n}$ velocity and $P$-wave velocities in the upper mantle. 


\section{REFERENCES}

1. Bullen, K. E., An Introduction to the Theory of Seismology, 3rd ed., (Cambridge University Press, 1963).

2. Dahlman, $\mathbf{O}$. and $\mathrm{H}$. Isratison, Monitoring Underground Nuclear Explosions, (Elsevier Scientific Publishing Company, Amsterdam, 1977).

3. Duclaux, F. and L. Michaud, "Conditions Experimentals des Tirs Nucleaires Souterrains Francais au Sahara, 1961-1966," C.R. Acad. Sc. Paris 27, Series B, 189-192 (1970).

4. Israelson, H., R. Slunga, and D: Dahlman, "Aftershocks Caused by the Novaya Zemlya Explosion on October 27, 1973," Nature 247, 450-452 (1974).

5. Marshall, P. D., D. L. Springer, and H. C. Rodean, "Magnitude Corrections for Attenuation in the Upper Mantle," Geophys. J. R. Astr. Soc. 57, 609-638 (1979).

6. Nordy!:e, M.D., "A Review of Soviet Data on the Peaceful Uses of Nuclear Explosions," Annals of Nuclear Frergy 2, 657-673 (Pergamon Press, New York, 1975).

7. Springer, D. L. and R. L. Kinnaman, "Seismic Suurce Summary for U.S. Underground Nuclear Explosions, 1961-1970," Bull. Seismol. Soc. Am. 61, 1073-1098 (1971).

8. Springer, D. L. and R. L. Kinnaman, "Seismic Source Summary for U.S. Underground Nuclear Explosions, 1971-1973," Eull. Seismol. Soc. Am. 65 , 343-349 (1975).

9. Volvovskii, I.C., Seismic Investigation of the Earth's Crust in the USSR, (Nedra Publishing House, Moscow, in Russian, 1973).

MLD/cg 